\title{
Regional Water Resources Security Grading Evaluation Considering Both Visible and Virtual Water: A Case Study on Hubei Province, China
}

\section{Yubo Du}

Wuhan University of Technology

Yan Tu ( $\nabla$ tuyan_belle@163.com )

Wuhan University of Technology

\section{Zongmin Li}

Sichuan University

\section{Ling Nie}

Chengdu University

\section{Research Article}

Keywords: Quantity of water resources, Pressure on water resources, Efficiency of water use, Water resources security evaluation, Fuzzy AHPSort II-EW method

Posted Date: October 5th, 2021

DOl: https://doi.org/10.21203/rs.3.rs-868644/v1

License: (9) This work is licensed under a Creative Commons Attribution 4.0 International License. Read Full License

Version of Record: A version of this preprint was published at Environmental Science and Pollution Research on December 1st, 2021. See the published version at https://doi.org/10.1007/s11356-02117506-2. 


\title{
Title Page
}

Title: Regional Water Resources Security Grading Evaluation Considering Both Visible and Virtual Water: A Case Study on Hubei Province, China

\section{Authors and affiliations}

\section{Yubo Du}

School of Safety Science and Emergency Management, Wuhan University of Technology, Wuhan 430070, China

\section{Yan Tu (Corresponding author) $\bowtie$}

School of Safety Science and Emergency Management, Wuhan University of Technology, Wuhan 430070, China E-mail: tuyan_belle@163.com; Tel: (86)-15207163276; Fax: (86-27)87859231

\section{Zongmin Li}

School of Business, Sichuan University, Chengdu 610065, China

\section{Ling Nie}

Business School, Chengdu University, Chengdu 610106, China

\begin{abstract}
The security of water resources is of great importance to long-term sustainability. In order to better ensure the security of water resources, a significant link is to conduct water resources security evaluation, which should be considered from many perspectives as it involves natural reserves, social production, the efficiency of use, and environmental protection. In this paper, a fuzzy Analytic Hierarchy Process Sort (AHPSort) II-entropy weight (EW) method for regional water resources security evaluation is proposed based on the security of visible water and virtual water. Firstly, this paper takes into account the criterion of efficiency of water use in addition to two other criteria of quantity of water resources, pressure on water resources to establish a comprehensive water resources security evaluation system. Secondly, a combination method of hesitant fuzzy language judgment and entropy weight is employed to obtain the weight of each indicator. Thirdly, AHPSort II is used to classify the security levels of the evaluated regions, in which the security levels of regional water resources are divided into five levels. Furthermore, a case study on the cities of Hubei Province, China is conducted to show the applicability of the proposed method, the effectiveness, and reliability of the method are then verified by being compared with a subjective method and an objective method as well as sensitivity analysis. Finally, according to the comprehensive evaluation results, specific management suggestions for improving the water resources security in the case are put forward.
\end{abstract}

\section{Keywords}

Quantity of water resources · Pressure on water resources · Efficiency of water use · Water resources security evaluation · Fuzzy AHPSort II-EW method

\section{Acknowledgments}

This research was supported by the National Natural Science Foundation of China (grant number 71801177), the Humanities and Social Sciences Fund of Ministry of Education of China (grant number 18YJC630163), and the Fundamental Research Funds for the Central Universities (grant number WUT: 2020VI006). 


\title{
- Regional Water Resources Security Grading Evaluation Considering Both Visible and Virtual Water: A Case Study on Hubei Province, China
}

${ }_{3}$ Yubo $\mathrm{Du}^{1} \cdot \operatorname{Yan} \mathrm{Tu}^{1} \cdot$ Zongmin $\mathbf{L i}^{2} \cdot \mathbf{L i n g} \mathrm{Nie}^{3}$

5 Received: date / Accepted: date

\begin{abstract}
The security of water resources is of great importance to long-term sustainability. In order to better ensure the security of water resources, a significant link is to conduct water resources security evaluation, which should be considered from many perspectives as it involves natural reserves, social production, the efficiency of use, and environmental protection. In this paper, a fuzzy Analytic Hierarchy Process Sort (AHPSort) II-entropy weight (EW) method for regional water resources security evaluation is proposed based on the security of visible water and virtual water. Firstly, this paper takes into account the criterion of efficiency of water use in addition to two other criteria of quantity of water resources, pressure on water resources to establish a comprehensive water resources security evaluation system. Secondly, a combination method of hesitant fuzzy language judgment and entropy weight is employed to obtain the weight of each indicator. Thirdly, AHPSort II is used to classify the security levels of the evaluated regions, in which the security levels of regional water resources are divided into five levels. Furthermore, a case study on the cities of Hubei Province, China is conducted to show the applicability of the proposed method, the effectiveness, and reliability of the method are then verified by being compared with a subjective method and an objective method as well as sensitivity analysis. Finally, according to the comprehensive evaluation results, specific management suggestions for improving the water resources security in the case are put forward.
\end{abstract}

Keywords Quantity of water resources · Pressure on water resources · Efficiency of water use · Water resources security evaluation · Fuzzy AHPSort II-EW method

\section{Introduction}

Water resources are not only the source of life but also important energy for social development. With the development of science, technology, and society, the security of water resources has been paid more and more attention, and the factors affecting the security of water resources have also become more and more. To be exact, with the rapid growth of the world's population, more and more countries are facing the problem of water quality and security (Cao et al. 2021). The insecurity of water resources will also cause many problems, so ensuring the security of water resources is the key to solve the problem at this time (Vorosmarty et al. 2010). Historically, water quantity has always been a problem that water shortage areas want to solve, and water quality is a problem that needs to be solved in water-rich areas. In today's society, the security of virtual water, that is, improving water efficiency, has become a major challenge facing the world. (Mans et al. 2016, Luo et al. 2020).

$\triangle$ Yan Tu

E-mail: tuyan_belle@163.com; Tel: (86)-15207163276; Fax: (86-27)87859231

1 School of Safety Science and Emergency Management, Wuhan University of Technology, Wuhan 430070, China

2 School of Business, Sichuan University, Chengdu 610065, China

3 Business School, Chengdu University, Chengdu 610106, China 
Vorosmarty et al. (2010) mentioned that 60 cities in the world will face serious water shortages by 2020 . According to statistics, China's average water resources rank the sixth in the world to $2.81 \times 10^{12} \mathrm{~m}^{3}$, but China's per capita water resources are only one-quarter of the world's water resources. According to the United Nations World Water Development Report 2019, global water consumption increases by 1\% every year. Given industrial and economic development, it is expected that by 2050 more than 2 billion people will live in countries with severe water shortages. About 4 billion people will suffer from severe water shortages for at least one month a year, and 22 countries will be at risk of severe water stress (The United Nations World Water Development Report 2019). According to China's 2015 State of the Environment Report, approximately 35.5\% of the statecontrolled river sections distributed in 10 large river basins across China contained water graded class IV, V, or worse, and is deemed unsafe for human consumption. Only $9.1 \%$ of groundwater monitoring sites distributed in 202 cities had good water quality, while $61.3 \%$ were deemed poor or worse (Ministry of Environmental Protection of China 2015). As shown by the above data, it is not difficult to see that there is a shortage of water resources and the pressure of pollution and use of water resources. At the same time, the distribution of water resources in many areas of China is unbalanced. Even in the areas with abundant water resources, it is particularly complicated to make the reasonable distribution of water resources. Therefore, the spatial imbalance between water supply and demand often occurs in China (Xu et al. 2020). As a large agricultural country, China has a lot of water resources used in agricultural irrigation. China's water resources are scarce and unevenly distributed, and the utilization efficiency of agricultural irrigation water is low. The effective utilization coefficient of farmland irrigation water in China is only 0.53, which is far lower than the average level of 0.80 in developed countries. Due to the increasing demand for water resources, the development of people's life and society and the security of water resources have become more closely related. Therefore, when evaluating the security of water resources, we often need to evaluate from many aspects and need to take indicators in different fields. In particular, countries are paying more and more attention to the security of virtual water, which is to materialize water resources in people's consumption and services ( $\mathrm{Xu}$ et al. 2020). This paper pays special attention to the efficiency of water use in water resources security, especially in areas with abundant water resource, to cope with the contradiction between rapid economic growth and the challenge of water use $(\mathrm{Fu}$ et al. 2021).

In the evaluation of water resources security, There are various methods of water resource security evaluation. For example, de Melo et al. (2021) established failure mode and impact analysis for water resources security analysis. Yin et al. (2020) established a water resources security evaluation model in the karst area according to fuzzy mathematics theory and made a dynamic evaluation on water resource security in the karst area on a certain time scale. Wang et al. (2020) used a projection pursuit model based on particle swarm optimization (PSO-PEE) to evaluate the water security of five Central Asian countries. Li et al. (2020) established a water resources security evaluation model to evaluate the water resources security situation in Guizhou Province. Ding et al. (2017) proposed a fuzzy comprehensive evaluation method based on the entropy weight method to evaluate the water environment security of the Heshan drinking water source area.

At the same time, when evaluating the security of regional water resources, it is necessary to classify the water resources security levels of each region for analysis, which is more convenient for decision-makers to make decisions. In previous studies, most multi-criteria decision making methods (MCDM) have been developed for selection and ranking problems where it can obtain the best alternatives for decision problems or rank their results based on indicators (Aguaron et al. 2019, Kang et al. 2019; Sun et al. 2020). Zhang et al. (2019) adopted TODIM (Portuguese acronym for interactive multi-criteria decision making) method to evaluate water security. As a basic decision-making method, AHP is widely used in various fields. However, it has a defect that when a decision has a large number of alternatives, it requires a large amount of calculation (Miccoli and Ishizaka 2017), however, the fuzzy AHPSort II-EW method can overcome this disadvantage very well (Miccoli and Ishizaka 2017; $\mathrm{Xu}$ et al. 2019). At the same time, comparison and sensitivity analysis are introduced into the fuzzy AHPSort II-EW method, which can improve the quality of decision results (Ishizaka and Lopez 2018). 

to these products for recycling and trading.

Fig. 1 Relationship between visible water and virtual water.

\section{Key problem statement}

\subsection{Water resources security}

It is a key step to get the weight of each indicator in AHPSort II, and the traditional method to calculate the weight is AHP (Sutadian et al. 2017). This paper uses the combination of the objective weight method and subjective weight method to obtain the weight of each indicator, in which the objective method is the entropy weight method and the subjective method is hesitant fuzzy language judgment. In particular, in the subjective method, an expert rating is required, so it is difficult to reach a consistent conclusion due to the different working experiences and educational background of each expert. Therefore, we could use the fuzzy hesitation language term set to represent the score of each expert ( $\mathrm{Li}$ et al. 2018), and consider the maximum consensus and minimum disagreement of all experts to build a model to obtain the weight of each expert (Zhang et al. 2019). To sum up, the results of the two weighting methods are combined and weighted and then combined with AHPSort II to form the fuzzy AHPSort II-EW method for regional water resources security evaluation.

The general structure of this paper is shown as follows: Key problem statement is given in Sect. 2. Evaluation methodology is mainly introduced in Sect. 3 including the overall framework, the introduction of various indicators, the introduction of subjective and objective weighting methods, and finally the introduction of AHPSort II. Sect. 4 is the case study, which includes data collection, data processing, calculation, classification of each city in Hubei Province, and comparative analysis and sensitivity analysis of the results. Finally, suggestions are put forward based on the results. The content of Sect. 5 is the conclusion of this paper, including the evaluation of the deficiencies of this paper and the outlook for the future.

This section describes each indicator and the source of each indicator in detail.

To put it bluntly, we can't live without water. Therefore, it is particularly important to ensure the security of water resources. In the past, people in areas with abundant water resource may ignore the importance of water resource security. However, as people pay more and more attention to the security of virtual water, water resource security in water-rich areas has become the focus. This paper considers the security of water resources based on visible water and virtual water. Visible water is very simple, that is, water resources that people can touch and see, and virtual water refers to the amount of water resources needed in the production of products and services, that is, the virtual water condensed in products and services (Chen et al. 2021). In other words, virtual water is an effective measure of water demand from the perspective of water consumption, that is, the number of water consumed in the process of production or service (Zhang et al. 2021).

Fig. 1 shows the relationship between visible water and virtual water (Sutadian et al. 2017). First of all, the most direct sources of visible water are atmospheric water, surface water, and groundwater. These three sources change periodically and transform into each other. Society uses precipitation and water diversion to form the relationship between circulating water supply and water use. In this process, water is also used to make different products, mainly industrial products, agricultural products, and energy products. Virtual water is also recycled

In this paper, in order to more fully consider the security of water resources, we will consider from three aspects, namely: quantity of water resources (Q) (Wang et al. 2020), pressure on water resources (P) (Tu et al. 2021) and efficiency of water use (E) ( $\mathrm{Li}$ et al. 2018, Song et al. 2017). Further explanations are as follows: Q means that the amount of water resources is at a level that can enable people's normal life and 
production activities; P means that water resources can withstand the pressure brought by population growth and environmental pollution; E means that water resources can be rationally used and distributed in various fields. Ensuring the efficiency of water use is also to ensure the security of virtual water resources. In today's world, more and more areas with abundant water resource are facing the problem of low use efficiency of water resources, and areas with abundant water resource are increasingly becoming the focus of ensuring virtual water security. The introduction of water resource efficiency indicator can more intuitively see the water resource risk in areas with abundant water resource and better solve the problem of water resource security. The reason why this paper chooses these three indicators is because: If we only consider the quantity and pressure on water resources, then we will waste a lot of water resources because there is no reasonable allocation of water resources; if we only start from the quantity and efficiency of water use, then we will also cause water pollution and ecological environment; if we only consider the efficiency of water use and the pressure on water resources, it will lead to a serious shortage of water resources or reduce productivity, which will affect the future of mankind. Therefore, in order to ensure the security of water resources and our sustainable development, these three criterions should be considered at the same time. Based on this idea, the water resource evaluation system in this paper is shown in Fig. 2,

Fig. 2 Regional water security evaluation system.

\subsection{Evaluation indicators}

As mentioned above, this paper divides water resources security into three criterions, and these three criterions are respectively expressed by some indicators, in the quantity of water resources including annual precipitation, surface water resources, groundwater resources, and water resources per capita, which are the key factors reflecting water security. The pressure on water resources include: density of population, wastewater emission, discharge into the river, which are the key factors to reflect the quality and security of water resources. The security indicators of water resources efficiency include water consumption per 10,000 yuan of GDP, water consumption per 10,000 yuan of industrial output value, water loss, and water consumption per mu for farmland irrigation, which are the key security factors of water resources efficiency. The table of water resources security indicator see in Table 1

Table 1 Water security evaluation indicators.

\begin{tabular}{|c|c|c|c|}
\hline Criterion & Indicator & Index type & Reference \\
\hline \multirow{4}{*}{ Quantity of water resources } & Annual precipitation $C_{11}$ & + & Ding et al. 2017 \\
\hline & Surface water resources $C_{12}$ & + & Mishra 2020 \\
\hline & Groundwater resources $C_{13}$ & + & de Graaf et al. 2019] \\
\hline & Water resources per capita $C_{14}$ & + & Ding et al. 2017); Zhao et al. 2021 \\
\hline \multirow{4}{*}{ Pressure on water resources } & Population density $C_{21}$ & - & Acuna-Alonso 2021]; Dou et al. 2021] \\
\hline & Wastewater discharge $C_{22}$ & - & Gusain et al. 2020 \\
\hline & Discharge into river $C_{23}$ & - & Abbott et al. 2019] \\
\hline & Water consumption per 10,000 yuan GDP $C_{31}$ & - & Wang et al. 2020 \\
\hline \multirow{3}{*}{ *Efficiency of water use } & Water consumption per 10,000 yuan of industrial added value $C_{32}$ & - & Ding et al. 2017) \\
\hline & Water loss $C_{33}$ & - & (Tu et al. 2021] \\
\hline & Average water consumption per mu for farmland irrigation $C_{34}$ & - & Liu et al. 2021; Zhao et al. 2021 \\
\hline
\end{tabular}

Note: "+" means benefit indicator, and "-" means cost indicator.

(1) With the increasing development and utilization of water resources, we represent the amount of natural water resources from three aspects: atmosphere, surface, and underground. And per capita water represent the water holding capacity of the society. 
(2) At present, the pollution of water resources mainly comes from the wastewater in life and industry. Therefore, the pressure of water resources quality is expressed as wastewater discharge and discharge into the river, and the pressure of water resource use is expressed as population density. It is worth noting that population density can reflect the population and also reflect the demand for water from one side. In this paper, population density is included in the pressure on water quality and water resources because the greater the demand, the greater the pressure on water resources, and the larger the population will also lead to the aggravation of water pollution from another side. So the pressure on water quality and water resources are expressed by these four indicators.

(3) Due to the low efficiency and uneven distribution of water resources in more and more water rich areas in the world, this paper considers the security of virtual water, which is closely related to the efficiency of water resources. The Water consumption per 10,000 yuan GDP and Water consumption per 10,000 yuan of industrial added value are common indicators to evaluate the efficiency of water resources. China as a large agricultural country, a large number of virtual water commodities are represented by food products. China's grain output is increasing year by year, largely because of the increasing irrigated area. The efficiency of irrigation water delivery in China is only $52 \%$, far lower than 70\%-80\% in developed countries (Kang et al. 2017). According to the (China Water Resources Bulletin 2019), irrigation water consumption accounted for 61.4 percent of the total water consumption of the national economy in 2019. The problems of traditional agricultural water resources, such as low irrigation water use coefficient, and large regional differences in water production efficiency, have not been fully solved. Therefore, it is necessary to take irrigation water consumption per mu as an indicator to evaluate water resources security. In today's society, a large part of the water is not renewable in daily production activities, and reducing such loss is also a manifestation of improving water use efficiency. Therefore, water loss is also an indicator to evaluate water use efficiency.

\subsection{Introduction of evaluation indicators}

Water resources security itself is a broad concept, which is related to its impact in various fields. In order to better express water resources security, this paper divides water resources security into three criterions, namely, quantity of water resources, pressure on water resources, and the efficiency of water use.

\subsubsection{Quantity of water resources}

Annual precipitation refers to the sum of precipitation in 12 months of a year, which can express the quantity of water resources in an area and is denoted as $C_{11}$.

Surface water resources refer to the dynamic water quantity of rivers, lakes, and other surface water bodies, that is, the runoff of natural rivers, which can be expressed as $C_{12}$. It is also an important indicator of the richness of water resources.

Groundwater resources (which can be expressed as $C_{13}$ ) refers to the dynamic amount of groundwater recharged by precipitation and infiltration of surface water (river, lake, and reservoir).

The amount of water resources per capita (expressed as $C_{14}$ ) indicates the abundance degree of social water resources. The total water resource is $W_{T T}$, and $C_{14}$ can be expressed as:

$$
C_{14}=\frac{W_{T T}}{P R}
$$

\subsubsection{Pressure on water resources}

In this paper, an indicator is used to express the pressure on water resources use in a region. The indicator is population density (which can be expressed as $C_{21}$ ), let $S$ denote the area of the region, the total population 
of the region is $P R$, and $C_{21}$ can be expressed as:

$$
C_{21}=\frac{P R}{S}
$$

In this paper, two indicators are used to express the pressure of water resources quality. One of them is the wastewater discharge $\left(C_{22}\right)$, which refers to the amount of water discharged by industrial $\left(W_{I}\right)$, tertiary industry $\left(W_{T}\right)$ and urban residents $\left(W_{P}\right)$, excluding the discharge of thermal power once-through cooling water and mine drainage.

$$
C_{22}=W_{I}+W_{T}+W_{P}
$$

Discharge into river is a relatively direct way to pollute water resources because people's daily living water is largely dependent on the use of the river, so the discharge into river is also an important indicator of water pollution (expressed by $C_{23}$ ).

\subsubsection{Efficiency of water resources}

Water resources and economic development is very close, the efficiency of water use is an indispensable part of water resources security, especially under the background that people pay more and more attention to virtual water security, water consumption of 10,000 yuan of GDP $\left(C_{31}\right)$ and Water consumption per 10,000 yuan of industrial added value $\left(C_{32}\right)$ can indirectly express the impact of water resources on the economy and whether the distribution of water resources is reasonable. Total water consumption is expressed as $W_{C}, 10,000$ yuan GDP as $G$, total industrial water consumption as $W_{I C}$, and the industrial output value of 10,000 yuan GDP as $O_{I}$, so $C_{31}$ and $C_{32}$ can be expressed as:

$$
C_{31}=\frac{W_{C}}{G}
$$

$$
C_{32}=\frac{W_{I C}}{O_{I}}
$$

Water loss $\left(C_{33}\right)$ is different from simple water consumption. Ordinary water consumption refers to the total amount of water used, while water consumption refers to the amount of water consumed by transpiration, evaporation, soil absorption, product taking away, residents and livestock drinking in the process of water conveyance and water consumption, that is, it can't return to any link in the water cycle. So this is an indicator of water use efficiency. Industrial water consumption is expressed as $I W$, agricultural water consumption is expressed as $A W$, and domestic water consumption is expressed as $S W, C_{33}$ can be expressed as:

$$
C_{33}=I W+A W+S W
$$

Agricultural products are the main virtual water products. In this paper, the average irrigation water consumption per mu $C_{34}$ refers to the water consumption per mu of farmland under the condition of meeting the normal yield of crops in the region. The smaller the water consumption is, the higher the utilization rate of water is, and the better the water distribution effect is. The total irrigation water volume of farmland is $A I$, the total farmland area is $A S, C_{34}$ can be expressed as:

$$
C_{34}=A I+A S
$$

\section{Evaluation methodology}

This section mainly introduces the framework of the research method used in this paper. 
3.1 Overall framework

The overall framework of this paper is as follows. There are $n$ evaluators, $E_{u}$ is $u$ th evaluator; there are $k$ indicators, $A_{k}(k=1,2, \ldots, p)$ is $k$ th indicator, the area to be evaluated is represented by $R$, and $R_{i}(i=$ $1,2, \ldots, r)$ is $i$ th area. In this study, hesitant fuzzy language term set is used to describe experts' judgment on the importance of indicators and the expert weight is calculated by a model that maximizes the consensus and minimizes the disagreement among experts. Then, by introducing the weighted average operator parameter, the indicator weight is calculated (which is the weight calculated by subjective method), and then the subjective weight is combined with the objective weight (entropy method) to get the weight of each indicator. Finally, the AHPSort II method is used to classify, and the final classification of each region is obtained. The overall block diagram of the evaluation method is shown in the Fig. 3 .

Fig. 3 The general framework of the evaluation methodology.

\subsection{Weight determination}

Since different indicators have different degrees of influence on water resource security, it is particularly important to select the weight of each indicator. In this paper, a combination of hesitant fuzzy language judgment (subjective method) and entropy weight method (objective method) is adopted to calculate the weight of each indicator.

\subsubsection{Hesitant fuzzy language judgment description}

Need to invite experts before we calculate subjective weight of each indicator scores, because the expert scoring is through language, this will lead to some inaccurate information has certain fuzziness, such as some experts for a can accurately know the evaluation indicator, and some experts may have no idea about this metric will draw a rough evaluation, Even some experts may never know this indicator and may not make evaluation, so we use hesitant fuzzy language term set to represent the scores of each expert, and the language is represented by numbers, and this method is more effective and more appropriate to people's real evaluation habits (Yan et al. 2016). Since each expert has different work experience and educational background, it is difficult for them to reach the same opinion ( $\mathrm{Li}$ et al. 2015). Therefore, we need to minimize the differences among experts and reach the maximum consensus.

This paper will use the language set of nine degrees to express the experts' evaluation, which can be expressed in the following:

$m=\left\{m_{\alpha} \mid \alpha=-z, \ldots,-1,0,1, \ldots, z\right\}$

$=\{$ extremely poor, very poor, poor, slightly poor, fair, slightly good, good, very good, extremely good $\}$

Use the numbers from 0 to 1 to represent the 9 graduations $\{0.0,0.125,0.25,0.375,0.5,0.625,0.75,0.875,1.0\}$ (Meng and Tang 2019, Luo et al. 2020). Let $F_{m}(I), I=1,2, \ldots, 5$ is denoted as a fuzzy restriction label. For example, five experts have evaluated the "groundwater resource" and the results are as follows: $F_{m}^{5}=$ $\left\{m_{-4}, m_{-3}, m_{-2}, m_{-1}, m_{0}\right\}=\{0.0,0.125,0.25,0.375,0.5\}, F_{m}^{4}=\left\{m_{1}, m_{2}, m_{3}, m_{4}\right\}=\{0.625,0.75,0.875,1.0\}, F_{m}^{3}$ $=\left\{m_{-3}, m_{-2}, m_{-1}\right\}=\{0.125,0.25,0.375\}, F_{m}^{1}=\left\{m_{1}\right\}=\{0.625\}, F_{m}^{2}=\left\{m_{2}, m_{3}\right\}=\{0.75,0.875\}$, as shown in Fig. 4 . 
It is not difficult to see that each expert's evaluation of the same indicator is not completely consistent. Therefore, in order to better process the data, we need to extend all language sets to the same length, that is, to increase the shorter language sets until all the language sets have the same length. The extension value is $\bar{h}=\mu h^{+}+(1-\mu) h^{-}$, where $\mu(0 \leq \mu \leq 1)$ is the degree of preference of experts, which can be divided into positive, negative, and neutral. Li et al. (2015) found that the weight of each language set extended by positive, negative and neutral attitude was almost the same, so this paper chose to extend each language set by neutral attitude, which indicates $\mu=1 / 2$, when experts are positive on elements, which indicates $\mu=1$, when negative $\mu=0, h^{+}$and $h^{-}$are the maximal and minimal values in $F_{m}(I)$, respectively. The extension example of hesitant fuzzy language term set can be seen in Table 2 In addition, if some experts have never known about the indicator, the blank set will appear, and then the evaluation results of other experts will be used to supplement it to make it consistent with the length of the hesitant fuzzy language term set of other experts.

Table 2 Extension example of hesitant fuzzy language term set.

\begin{tabular}{lr}
\hline Original hesitant fuzzy language term set & \multicolumn{1}{c}{ Extension } \\
\hline$F_{m}^{5}=\{0.0,0.125,0.25,0.375,0.5\}$ & $\{0.0,0.125,0.25,0.375,0.5\}$ \\
$F_{m}^{4}=\{0.625,0.75,0.875,1.0\}$ & $\{0.625,0.75,0.8125,0.875,1.0\}$ \\
$F_{m}^{3}=\{0.125,0.25,0.375\}$ & $\{0.125,0.25,0.25,0.25,0.375\}$ \\
$F_{m}^{1}=\{0.625\}$ & $\{0.625,0.625,0.625,0.625,0.625\}$ \\
$F_{m}^{2}=\{0.75,0.875\}$ & $\{0.75,0.8125,0.8125,0.8125,0.875\}$ \\
\hline
\end{tabular}

\subsubsection{Expert weight determination}

In decision-making problems in daily life, we can judge according to our own views on the problem. However, in the multi-objective decision problem, the function of this judgment is left to the experts to decide, and we have mentioned above that it is more appropriate to use the hesitant fuzzy language term set to represent the evaluation of the experts. In this paper, a model will be used to minimize the disagreement of each expert and maximize the consensus of each expert to find the best expert weight. The ambiguity of an indicator can be expressed by the hesitation of experts, while the differences between experts can be expressed by the Euclidean distance.

First of all, as mentioned above, each expert uses hesitant fuzzy language to evaluate the importance of each indicator. The hesitant fuzzy language can be transformed into corresponding hesitant fuzzy numbers, and each hesitant fuzzy language term set can be extended to the same length $L$, which can be expressed as the following formula:

$$
y_{u k}=\left\{y_{u k}^{l} \mid l=1,2, \ldots, L ; u=1,2, \ldots, n ; k=1,2, \ldots, p\right\}
$$

where $L$ means the length of $y_{u k}$.

In order to get the maximum degree of consensus and the minimum degree of disagreement among experts, we need to find the best expert weight which can be noted as $\omega_{u}^{E}(u=1,2, \ldots, n)$, so the sum of all fuzzy hesitation should be the minimum, and the difference of all fuzzy evaluation with expert weight should be the minimum. According to the above analysis, an optimization model is established to minimize the judgment 
deviation and hesitation between evaluation results. This model is shown below:

$$
\begin{aligned}
& \min \sum_{k=1}^{p}[\bar{d}(y) \times \bar{f}(\phi y)] \\
&=\sum_{k=1}^{p} \sqrt{\frac{1}{L} \sum_{l=1}^{L} \sum_{u=1}^{n} \sum_{x=1, n \neq x}\left(\omega_{u}^{E} y_{u k}^{l}-\omega_{x}^{E} y_{x k}^{l}\right)^{2}} \times \sqrt{\sum_{u=1}^{n} \sum_{x=1, u \neq x}\left(\omega_{u}^{E} \phi y_{u k}-\omega_{x}^{E} \phi y_{x k}\right)^{2}} \\
& \text { s.t. }\left\{\begin{array}{l}
y_{u k}=\left\{y_{u k}^{l} \mid l=1, \ldots, L, u=1, \ldots, n, k=1, \ldots p\right\} \\
y_{x k}=\left\{y_{x k}^{l} \mid l=1, \ldots L, x=1, \ldots, n, k=1, \ldots, p, u \neq x\right\} \\
\sum_{u=1}^{n} \omega_{u}^{E}=1 \\
\omega_{x}^{E} \geq o, u=1, \ldots, n
\end{array}\right.
\end{aligned}
$$

where $\omega_{u}^{E}$ represents the weight of $E_{u}, \sqrt{\frac{1}{L} \sum_{l=1}^{L} \sum_{u=1}^{n} \sum_{x=1, u \neq x}^{n}\left(\omega_{u}^{E} y_{u k}^{l}-\omega_{x}^{E} y_{x k}^{l}\right)^{2}}$ is the weighted sum of Euclidean distance between one expert and another expert for indicator $A_{k}, f(\phi y)=\sqrt{\sum_{u=1}^{n} \sum_{x=1, u \neq x}\left(\phi y_{u k}-\phi y_{x k}\right)^{2}}$ is the difference of hesitancy degree between the two experts for indicator $A_{k}, \phi y_{u k}$ is the hesitancy degree of hesitant fuzzy elements and $y_{u k}$ is the fuzzy hesitant element.

Because of the hesitation degree and Euclidean distance of the average judgment score among experts in Eq. 9 are the smallest, so it can solve the best $\omega_{u}^{E}(u=1,2, \ldots, n)$.

\subsubsection{Indicators' weights computation}

According to Sect. 3.2.2, each expert's hesitant fuzzy language term set has been extended to the same length which has been extended to the same length and the expert weight can be obtained. Next, the weight of each indicator can be calculated according to the expert weight. Therefore, we need to introduce the parameters of the weighted average operator, which is shown as follows:

$$
\bar{\gamma}_{k}=\sum_{u=1}^{n} \bar{y}_{u k}^{1}
$$

$$
\bar{\zeta}_{k}=\sum_{u=1}^{n} \frac{1}{L-2}\left(\bar{y}_{u k}^{2}+\bar{y}_{u k}^{3}+\ldots+\bar{y}_{u k}^{L-1}\right)
$$

$$
\bar{\delta}_{k}=\sum_{u=1}^{n} \bar{y}_{u k}^{L}
$$

The triangular fuzzy number $\left(\bar{\gamma}_{k}, \bar{\zeta}_{k}, \bar{\delta}_{k}\right)$ can be obtained from Eqs. 10p 12, which are similar to the de-fuzzification of an intuitionistic fuzzy numbers. The importance of the $k$ th indicator can be calculated by using the weighted average operator.

$$
\omega_{k}^{F}=\frac{\bar{\gamma}_{k}+\bar{\delta}_{k} \times\left(\frac{\bar{\gamma}_{k}}{\bar{\gamma}_{k}+\overline{\zeta k}}\right)}{\sum_{k=1}^{p}\left[\bar{\gamma}_{k}+\bar{\delta}_{k} \times\left(\frac{\bar{\gamma}_{k}}{\bar{\gamma}_{k}+\bar{\zeta} k}\right)\right]^{\prime}}
$$

Through the above process, we can get the indicator weight obtained by the hesitant fuzzy language judgment.

The above method is the hesitant fuzzy language judgment, which has strong subjectivity and is influenced by the background and knowledge of experts. The entropy weight method is an objective method to calculate the weight of indicators. Combining the entropy method with the hesitant fuzzy language judgment can better complement each other and improve the reliability of the results. So this paper combines the two methods to get the indicator weight. The following is the calculation process of the entropy weight method (Liu et al. 2021):

Step 1. Assuming that the original data matrix $X$ :

$$
X=\left(X_{i k}\right)_{r \times p}
$$

Step 2. Then data matrix $X$ is converted into the normalized matrix nor as follows: 
(1) Normalized matrix of benefit indicators:

$$
\alpha_{i k}=\frac{x_{i k}-\min \left(x_{i k}\right)}{\max \left(x_{i k}\right)-\min \left(x_{i k}\right)}, i=1,2, \ldots, r ; k=1,2, \ldots, p
$$

(2) Normalized matrix of cost indicators:

$$
\alpha_{i k}=\frac{\max \left(x_{i k}\right)-x_{i k}}{\max \left(x_{i k}\right)-\min \left(x_{i k}\right)}, i=1,2, \ldots, r ; k=1,2, \ldots, p
$$

Step 3. Calculate the entropy value of the $k$ th indicator:

$$
\epsilon_{k}=-\frac{1}{\ln r} \sum_{i=1}^{r} \beta_{i k} \ln \beta_{i k}, k=1,2, \ldots, p
$$

where, if $\beta_{i k}=0$ then $\beta_{i k} \ln \beta_{i k}=0$ and

$$
\beta_{i k}=\frac{x_{i k}}{\sum_{i=1}^{r} x_{i k}}, i=1,2, \ldots, r ; k=1,2, \ldots, p
$$

Step 4. Calculate the weight of the indicator as is as below:

$$
\omega_{k}^{S H}=\frac{1-\epsilon_{k}}{p-\sum_{k=1}^{p} \epsilon_{k}}, k=1,2, \ldots, p
$$

Based on the results of the above two weight methods, they are combined and weighted to obtain the final weight of the $k$ th indicator, which is expressed by the following formula:

$$
\omega_{k}^{A}=(1-V) \times \omega_{k}^{F}+V \times \omega_{k}^{S H}, k=1,2, \ldots, p
$$

where, $V$ represents the proportion of the weight of entropy weight method in the total weight, and $1-V$ represents the proportion of the hesitant fuzzy language judgment in the total weight. On the premise of generality, the value of $V$ is set to 0.5 .

\subsection{AHPSort II based aggregation method}

AHPSort II is a modification based on AHPSort, which uses AHP to calculate the weight. In this paper, the weight of each indicator is the combined weight. In this way, the combination of objective and subjective methods is more reliable than the simple use of a subjective method, so it's a little bit different than it was originally, and some changes are mainly reflected in the weight calculation, the steps are as follows (Miccoli and Ishizaka 2017; Ishizaka and Lopez 2018):

Step 1. Determine the objectives and problems to be studied, the indicator $A_{k}, k=1,2, \ldots, p$, and the alternative $R_{i}, i=1,2, \ldots, r$.

Step 2. Define the classification $Z_{w}, w=1,2, \ldots, W$, where $W$ represents the number of categories, and such categories have a certain order of good and bad. For example, 1 corresponds to good, 2 corresponds to not bad, etc.

Step 3. Determine the number of clusters in an indicator and denote it by $S_{j}, j=1,2, \ldots, M$.

Step 4. Define the outline of each class. It can be implemented with a local limit profile $l p_{j k}$, which is the minimum indicator required to represent each standard $k$ belonging to $S_{j}$ class. This feature is given by a typical example based on the elements of $S_{j}$ class belonging to standard $k$ noted as $S_{j k}$. We need a $k(M-1)$ constraint profile to define each class.

Step 5. Using the method of calculating the weight described above, the weight of the indicator is calculated, which is expressed as $\omega_{k}$.

Step 6. In each indicator $k$, the representative point $S_{j k}^{o}(o=1,2, \ldots, O)$ uniformly distributed in each indicator is selected. The limiting profile in this paper are selected by experts according to the original data 
after standardization. Experts' selection of representative points is an important step in AHPSort II. Different representative points will get different results, so the selection of representative points needs to be cautious. Therefore, we use the following method to select representative points:

First of all, we need to determine the number of representative points $\left(N_{S}\right)$ and the number of clusters $\left(N_{Q}\right.$, which will be introduced in Sect. 3.3.1 in an indicator.

$$
\frac{N_{Q}}{N_{S}}=B>1
$$

where $\mathrm{B}$ is a constant determined by the decision-maker. The larger B is, the higher the accuracy is.

Because the data are standardized, so the minimum is 0 , the maximum is 1 . Therefore, each representative point can be calculated by the following formula.

For the first cluster in each indicator, that is, the cluster starting from 0 , the representative points are calculated by the following formula:

$$
S_{M k}^{o}=\frac{l p_{M-1 k}}{N_{S}} \times o-1, o=1,2, \ldots, O
$$

especially, $S_{M k}^{1}=0$

The calculation method of representative points in the cluster from the last to 1 is as follows:

$$
S_{1 k}^{o}=l p_{1 k}+\frac{1-l p_{1 k}}{N_{S}} \times o, o=1,2, \ldots, O
$$

especially, $S_{M k}^{o}=1$

The solution of the representative points in the middle cluster $(1<j<M)$ is as follows:

$$
S_{j k}^{o}=l p_{j k}+\frac{l p_{j-1 k}-l p_{j k}}{N_{S}+1} \times o, o=1,2, \ldots, O
$$

Step 7. The representative points and limiting profiles in two paired matrices are compared. In this process, clustering can be used to reduce computation. Starting from the comparison matrix, the local priority $P_{o k}$ of the representative point and the local priority $P_{j k}$ of the limiting profile can be obtained by the eigenvalue method in Eq. (25).

$$
a \times P=\lambda \times P
$$

where $a$ is the comparison matrix; $P$ is the priorities weight vector; $\lambda$ is the maximal eigenvalue.

Step 8. If alternative $R_{i}$ is between two consecutive representative points $S_{o k}$ and $S_{o+1 k}$, we can get the local priority $P_{i k}$, which can be expressed by the following formula:

$$
P_{i k}=P_{o k}+\frac{P_{o+1 k}-P_{o k}}{S_{o+1 k}-S_{o k}} \times\left(T_{k}\left(R_{i}\right)-S_{o k}\right)
$$

where $S_{o k}$ and $S_{o+1 k}$ are two continuous representative points on indicator $k ; P_{o k}$ and $P_{o+1 k}$ are the local priorities of two continuous representative points on indicator $k ; T_{k}\left(R_{i}\right)$ is the score of the alternative $R_{i}$ on indicator $k ; P_{i k}$ is the local priority of $R_{i}$.

Step 9. Adding the weighted local priorities is the global priority $P_{i}$ of the alternative $i$. The global priority $l p_{j}$ of the limiting profile can be expressed by the following formula:

$$
\begin{aligned}
P_{i} & =\sum_{k=1}^{p} P_{i k} \times \omega_{k} \\
l p_{j} & =\sum_{k=1}^{p} \omega_{k} \times P_{j k}
\end{aligned}
$$

The comparison of $P_{i}$ and $l p_{j}$ is used to assign alternatives to class $A_{j}$.

If the limiting profile has been defined, alternative $R_{i}$ is assigned to the class $A_{j}$ which has an $l p_{j}$ just below the global priority $P_{i}$ (see Fig. 5).

$$
P_{i} \geq l p_{1} \rightarrow R_{i} \in Q_{1} ; l p_{2} \leq P_{i}<l p_{1} \rightarrow R_{i} \in Q_{2} ; \ldots ; P_{i}<l p_{M-1} \rightarrow R_{i} \in Q_{M}
$$


Fig. 5 Sorting with limiting profiles.

Step 10. Repeat Step 5 to Step 9 for each alternative that needs to be classified.

Step 11. Because AHPSort II uses the linear approximation method, it is necessary to fine-tune the results and check the selected scheme above and below the limiting profile to obtain an accurate classification.

\subsubsection{Clustering method}

This clustering method is mainly used to reduce computation

Step 1. The representative points and limiting profile in each indicator are selected.

Step 2. Representative points and limiting profiles are divided into clusters. The last compared element becomes the connection point of the two cluster boundaries can be seen in Fig. 6. Assuming that the number of representative points is 5 , there are 3 limiting profile, and they are divided into three clusters. The local priority is calculated from these 3 clusters.

Fig. 6 Example of clusters.

Step 3. Compare the clustering elements in the matrix and calculate the local priority of each cluster. The priorities of the cluster are connected by a common element that is also used to convert the indicator of the next cluster to the indicator of the previous cluster, making it computable one by one.

\section{Case study}

The water resources security evaluation method based on fuzzy AHPSort II-EW method is applied here to Hubei Province, including all municipal administrative units under the jurisdiction of Hubei Province.

\subsection{Case description}

Hubei is located in the middle reaches of the Yangtze River and north of Dongting Lake in Central China. It is known as the "Province of a thousand lakes". The volume of surface water is the fourth largest in China. From the above description, everyone will think that Hubei has developed water system, dense water network, and numerous lakes. It must be a province rich in water resources. However, this is not the case. According to the statistics, Hubei water resources account for $3.5 \%$ of the country, ranking tenth, with an average per capita share of 1,731 cubic meters, ranking seventeenth, accounting for only $73 \%$ of the national per capita share, close to the internationally recognized warning line of 1,700 cubic meters of serious water shortage.

We can give an example about the pollution of water resources in Hubei Province. The East Lake in Wuhan is the largest lake in Chinese cities, six times the size of the West Lake (Hangzhou, China). Although the water pollution control of the East Lake has spent a lot of money, the water quality of the East Lake is very poor and is not suitable for direct human contact. Therefore, the quality protection of water resources is also an urgent problem to be solved.

In terms of water resources utilization, although the overall water supply in Hubei Province is sufficient, due to the uneven distribution of water resources, coupled with climate change and the growing demand for water, the problem of water resources shortage in some areas of Hubei Province has become increasingly prominent in some periods. When precipitation is concentrated, flood is the main problem, and when precipitation is less, local drought and water shortage. And the efficiency of agricultural water use in Hubei Province is also very low. In this case, it is key to establish an appropriate water resources evaluation system. It is also urgent to put forward some suggestions to solve the efficiency of water resources use. 
The above three problems just reflect the applicability of the water resource security evaluation system in this paper. Hubei Province, as a typical province with abundant water resources but low water use efficiency, can analyze some deficiencies existing in various regions of Hubei Province by combining the security evaluation of visible water and virtual water.

\subsection{Data collection}

The data of this paper are collected from the National Bureau of Statistics, the water resources bulletin of Hubei Province and the official websites of various cities (National Bureau of statistics 2019, Hubei Water Resources Bulletin in 2019). The collected indicators are annual precipitation, surface water resources, groundwater resources, Water resources per capita, population density, wastewater discharge, Discharge into river, water consumption per 10,000 yuan of GDP, water consumption per 10,000 yuan of industrial added value, water loss, water consumption per mu of farmland irrigation. The data corresponding to the above indicators are the data of all cities in Hubei Province in 2019. The selection of indicator data strictly follows the principle of operability, and each indicator is added according to other articles or practical problems.

\subsection{Result interpretation}

In this paper, the indicator data of water resources security of cities in Hubei Province are shown in Table 3 (all data are in 2019). In the process of calculating the indicator weight, a subjective weight method and an objective weight method are used to combine the weights, so the indicator weights of the two methods are calculated separately. In the method of subjective weight, it is necessary to ask experts to score each indicator with fuzzy language. So five experts on water resources security are invited to evaluate the importance of the indicators in this paper. Each expert uses his past work experience and background to score the indicators by using fuzzy language. The scoring results are shown in Table 4 . Then, the scoring results of each expert are extended from different to the same length, and Lingo software is used to calculate Eq. (9) to get the weight of each expert, and then the weight of each indicator $\left(\omega_{k}^{F}\right)$ is calculated by Eqs. $10 \sim(12)$. Next, the entropy weight method is used to calculate the weight of each indicator to get the subjective weight $\left(\omega_{k}^{S}\right)$, both $\omega_{k}^{F}$ and $\omega_{k}^{S}$ can be seen in Table 5 . Finally, the final weight of each indicator is obtained by combining the weights calculated by the two methods, which are shown in Table 6

Table 3 Water resources security indicator data of Hubei province in 2019.

\begin{tabular}{|c|c|c|c|c|c|c|c|c|c|c|c|}
\hline \multirow{2}{*}{ City } & \multicolumn{4}{|c|}{ Quantity of water resources } & \multicolumn{3}{|c|}{ Pressure on water resources } & \multicolumn{4}{|c|}{ Efficiency of water use } \\
\hline & $C_{11}\left(\mathrm{~m}^{3} / 10^{8}\right)$ & $C_{12}\left(\mathrm{~m}^{3} / 10^{8}\right)$ & $C_{13}\left(m^{3} / 10^{8}\right)$ & $C_{14}\left(m^{3}\right)$ & $C_{21}\left(\mathrm{~m}^{3} /\right.$ person $)$ & $C_{22}\left(\operatorname{ton} / 10^{4}\right)$ & $C_{23}\left(\operatorname{ton} / 10^{4}\right)$ & $C_{31}\left(m^{3}\right)$ & $C_{32}\left(m^{3}\right)$ & $C_{33}\left(m^{3} / 10^{8}\right)$ & $C_{34}\left(m^{3}\right)$ \\
\hline Wuhan & 84.71 & 30.34 & 9.73 & 301 & 1319.991 & 88608 & 62026 & 23 & 35 & 12.44 & 320 \\
\hline Huangshi & 50.61 & 22.03 & 6.63 & 938 & 539.3192 & 28087 & 19660 & 107 & 149 & 4.65 & 432 \\
\hline Shiyan & 195.88 & 57.79 & 23.47 & 1701 & 143.4966 & 28346 & 19843 & 44 & 33 & 4.08 & 362 \\
\hline Yichang & 190.89 & 65.01 & 32.28 & 1594 & 196.2578 & 41853 & 29299 & 36 & 30 & 7.74 & 305 \\
\hline Xiangyang & 121.33 & 20.53 & 15.64 & 446 & 287.9157 & 50231 & 35162 & 68 & 58 & 13.1 & 386 \\
\hline Ezhou & 17.96 & 6.85 & 2.13 & 769 & 664.8055 & 12454 & 8718 & 140 & 241 & 2.81 & 345 \\
\hline Jinmen & 88.79 & 15.66 & 9.89 & 578 & 233.594 & 30802 & 21562 & 106 & 47 & 10.78 & 339 \\
\hline Xiaogan & 63.06 & 16.82 & 6.21 & 369 & 552.3008 & 33459 & 23423 & 120 & 95 & 11.25 & 311 \\
\hline Jinzhou & 133.01 & 46.89 & 14.99 & 956 & 395.9693 & 49646 & 34754 & 150 & 59 & 10.78 & 288 \\
\hline Huanggang & 174.51 & 67.74 & 20.48 & 1108 & 362.7771 & 45749 & 32024 & 124 & 52 & 14.3 & 376 \\
\hline Xianning & 125.66 & 65.66 & 14.87 & 2684 & 253.5974 & 32270 & 22589 & 91 & 68 & 7.12 & 380 \\
\hline Suizhou & 54.87 & 6.97 & 2.4 & 314 & 230.4898 & 17168 & 12019 & 82 & 30 & 4.58 & 340 \\
\hline Enshi & 265.31 & 124.92 & 44.13 & 3685 & 140.6639 & 14934 & 10453 & 45 & 34 & 2.58 & 237 \\
\hline Xiantao & 24.51 & 9.91 & 2.6 & 996 & 449.212 & 14130 & 9891 & 119 & 44 & 5.42 & 457 \\
\hline Qianjiang & 17.53 & 5.93 & 1.89 & 763 & 482.0858 & 12208 & 8546 & 98 & 42 & 4.03 & 436 \\
\hline Tianmen & 20.72 & 5.5 & 3.03 & 585 & 475.7437 & 11068 & 7748 & 143 & 35 & 4.87 & 414 \\
\hline Shennongjia & 31.63 & 14.85 & 6.94 & 19508 & 23.35789 & 701 & 491 & 52 & 96 & 0.0 & 402 \\
\hline
\end{tabular}


Table 4 Expert fuzzy judgments to the importance of indicators.

\begin{tabular}{|c|c|c|c|c|c|}
\hline Indicator & $E_{1}$ & $E_{2}$ & $E_{3}$ & $E_{4}$ & $E_{5}$ \\
\hline$C_{11}$ & $(0.5,0.625)$ & $(0.875)$ & $(0.75)$ & $(0.25,0.375,0.5)$ & $(0.75)$ \\
\hline$C_{12}$ & $(0.625,0.75,0.875)$ & $(0.5,0.625,0.75)$ & $(0.375,0.5)$ & $(0.375)$ & $(0.5,0.625)$ \\
\hline$C_{13}$ & $(0.875)$ & $(0.625,0.75,0.875)$ & $(0.625)$ & $(0.5,0.625,0.75)$ & $(0.875)$ \\
\hline$C_{14}$ & $(0.125,0.25,0.375)$ & $(0.5)$ & $(0.375,0.5)$ & $(0.75)$ & $(0.25)$ \\
\hline$C_{21}$ & $(0.875,1)$ & $(0.75,0.875)$ & $(0.75)$ & $(0.625,0.75)$ & $(0.5,0.625,0.75,0.875,1)$ \\
\hline$C_{22}$ & $(0.375,0.5,0.625)$ & $(0.625)$ & $(0.25,0.375,0.5)$ & $(0.375,0.5)$ & $(0.625,0.75)$ \\
\hline$C_{23}$ & $(0.5,0.625,0.75,0.875,1)$ & $(0.375,0.5)$ & $(0.5,0.625)$ & $(0.125,0.25,0.375)$ & $(0.75)$ \\
\hline$C_{31}$ & $(0.25,0.375)$ & $(0.375,0.5)$ & $(0.125,0.25,0.375)$ & $(0.625,0.75)$ & $(0.375,0.5)$ \\
\hline$C_{32}$ & $(0.75)$ & $(0.5,0.625)$ & $(0.5)$ & $(0.375,0.5,0.625)$ & $(0.375)$ \\
\hline$C_{33}$ & $(0.75,0.875)$ & $(0.375,0.5,0.625)$ & $(0.5,0.625)$ & $(1)$ & $(0.625,0.75)$ \\
\hline$C_{34}$ & $(0.375,0.5)$ & $(0.125,0.25,0.375,0.5,0.625)$ & $(0.625,0.75,0.875)$ & $(0.5,0.625)$ & $(0.375,0.5,0.625)$ \\
\hline
\end{tabular}

Table 5 The weights of HFLJ and EWM.

\begin{tabular}{llllllllllll}
\hline Method & $C_{11}$ & $C_{12}$ & $C_{13}$ & $C_{14}$ & $C_{21}$ & $C_{22}$ & $C_{23}$ & $C_{31}$ & $C_{32}$ & $C_{33}$ & $C_{34}$ \\
\hline HFLJ & 0.104 & 0.081 & 0.116 & 0.12 & 0.08 & 0.08 & 0.071 & 0.11 & 0.076 & 0.078 & 0.08 \\
EWM & 0.117 & 0.15 & 0.139 & 0.331 & 0.02 & 0.024 & 0.024 & 0.067 & 0.02 & 0.054 & 0.053 \\
\hline
\end{tabular}

Note: HFLJ represents hesitant fuzzy language judgment, EWM represents entropy weight method.

Table 6 Weight of each indicator.

\begin{tabular}{lc}
\hline \multicolumn{1}{c}{ Indicator } & Weight \\
\hline Annual precipitation $\left(C_{11}\right)$ & 0.111 \\
Surface water resources $\left(C_{12}\right)$ & 0.116 \\
Groundwater resources $\left(C_{13}\right)$ & 0.128 \\
Water resources per capita $\left(C_{14}\right)$ & 0.225 \\
Population density $\left(C_{21}\right)$ & 0.05 \\
Wastewater discharge $\left(C_{22}\right)$ & 0.053 \\
Discharge into river $\left(C_{23}\right)$ & 0.047 \\
Water consumption per 10,000 yuan GDP $\left(C_{31}\right)$ & 0.089 \\
Water consumption per 10,000 yuan of industrial added value $\left(C_{32}\right)$ & 0.048 \\
Water loss $\left(C_{33}\right)$ & 0.066 \\
Average water consumption per mu for farmland irrigation $\left(C_{34}\right)$ & 0.066 \\
\hline
\end{tabular}

After calculating the weight of each indicator, we can use the method of AHPSort II to classify, AHPSort II is a method evolved from AHP, which is based on the upgrade of AHP and reduces the computation to a great extent. In this paper, the water resources security of Hubei Province is divided into five levels: low risk, medium-low risk, medium risk, medium-high risk, and high risk which are noted as I, II, III, IV, and V. Before classification, experts should select the limiting profiles according to the original data after each indicator has been standardized, and then use Eqs. (22) $\sim(24)$ to calculate the representative points. The limiting profiles can be seen in Table 7, then each indicator are divided into different clusters, which is a vital step in AHPSort II. Table 8 shows the representative points and limiting conditions of annual precipitation. The annual precipitation is divided into two clusters, and the eigenvalue method is used to calculate the matrix. Table 9 and Table 10 show the weight of two clusters of annual precipitation calculated by the eigenvalue method. The two groups of weights are converted by the joint point. multiplying them by the ratio of the scores of joining point 0.4 in the two clusters: $0.3474 / 0.0337=10.3086$. Normalized local priorities of the representative points and the limiting profiles for the annual precipitation can be seen in Table 11.

In the alternative, the local priority $s$ of annual precipitation in Yichang can be calculated by Eq. 26), in which the annual precipitation in Yichang is $T_{k}\left(R_{i}\right)=0.6997$ (standardized data), $S_{o+1 k}=0.75, S_{o k}=0.5$, $P_{o+1 k}=0.6336, P_{o k}=0.3536$.

$$
P_{i k}=0.3536+\frac{0.6335-0.3536}{0.75-0.5} \times(0.6997-0.5)=0.5772
$$


Table 7 Limiting profile.

\begin{tabular}{|c|c|c|c|c|c|c|c|c|c|c|c|}
\hline \multirow{2}{*}{ Indicator } & \multicolumn{2}{|c|}{ Values of alternatives } & \multicolumn{9}{|c|}{ Class of risk } \\
\hline & Min & Max & High & $l p_{1}$ & Medium-High & $l p_{2}$ & Medium & $l p_{3}$ & Medium-low & $l p_{4}$ & Low \\
\hline annual rainfall & 17.53 & 265.31 & $<0.05$ & 0.05 & $0.05-0.2$ & 0.2 & $0.2-0.4$ & 0.4 & $0.4-0.5$ & 0.5 & $0.5<$ \\
\hline surface water resources & 5.5 & 124.92 & $<0.05$ & 0.05 & $0.05-0.2$ & 0.2 & $0.2-0.35$ & 0.35 & $0.35<0.6$ & 0.6 & $0.6<$ \\
\hline groundwater resources & 1.89 & 44.13 & $<0.11$ & 0.11 & $0.11-0.2$ & 0.2 & $0.2-0.3$ & 0.3 & $0.3-0.5$ & 0.5 & $0.5<$ \\
\hline water resources per capita & 301 & 19508 & $<0.01$ & 0.01 & $0.01-0.025$ & 0.025 & $0.025-0.04$ & 0.04 & $0.04-0.1$ & 0.1 & $0.1<$ \\
\hline population density & 23.3579 & 1319.991 & $<0.6$ & 0.6 & $0.6-0.7$ & 0.7 & $0.7-0.8$ & 0.8 & $0.8<0.9$ & 0.9 & $0.9<$ \\
\hline wastewater discharge & 701 & 88608 & $<0.5$ & 0.5 & $0.5-0.65$ & 0.65 & $0.65-0.8$ & 0.8 & $0.8<0.85$ & 0.85 & $0.85<$ \\
\hline discharge into river & 491 & 62026 & $<0.5$ & 0.5 & $0.5-0.65$ & 0.65 & $0.65-0.8$ & 0.8 & $0.8<0.85$ & 0.85 & $0.85<$ \\
\hline $\begin{array}{l}\text { water consumption per } 10,000 \\
\text { yuan GDP }\end{array}$ & 23 & 150 & $<0.2$ & 0.2 & $0.2-0.4$ & 0.4 & $0.4-0.8$ & 0.8 & $0.8<0.85$ & 0.85 & $0.85<$ \\
\hline $\begin{array}{l}\text { water consumption per } 10,000 \\
\text { yuan of industrial added value }\end{array}$ & 30 & 241 & $<0.7$ & 0.7 & $0.7-0.9$ & 0.9 & $0.9-0.95$ & 0.95 & $0.95-0.98$ & 0.98 & $0.98<$ \\
\hline Water loss & 0.07 & 14 & $<0.2$ & 0.2 & $0.2-0.4$ & 0.4 & $0.4-0.7$ & 0.7 & $0.7-0.8$ & 0.8 & $0.8<$ \\
\hline $\begin{array}{l}\text { average water consumption per } \\
\text { mu for farmland irrigation }\end{array}$ & 237 & 457 & $<0.2$ & 0.2 & $0.2-0.4$ & 0.4 & $0.4-0.6$ & 0.6 & $0.6-0.7$ & 0.7 & $0.7<$ \\
\hline
\end{tabular}

Table 8 Representative points and limiting profiles of "annual precipitation".

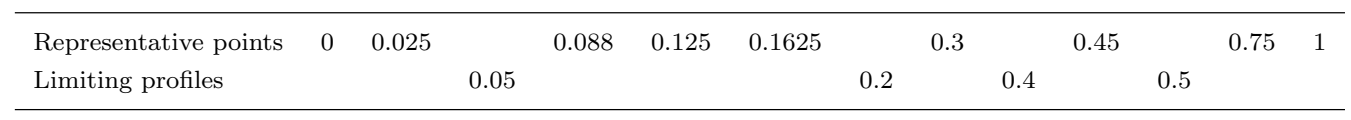

Table 9 The first cluster "annual precipitation".

\begin{tabular}{|c|c|c|c|c|c|c|c|c|c|}
\hline & & 0 & 0.025 & $l p_{1}(0.05)$ & 0.088 & 0.125 & 0.1625 & $l p_{2}(0.2)$ & Local priority \\
\hline \multirow{6}{*}{$l p_{1}$} & 0 & 1 & $1 / 2$ & $1 / 5$ & $1 / 3$ & $1 / 2$ & $1 / 3$ & $1 / 7$ & 0.0403 \\
\hline & 0.025 & 2 & 1 & $1 / 3$ & $1 / 3$ & $1 / 3$ & $1 / 3$ & $1 / 3$ & 0.0568 \\
\hline & 0.05 & 5 & 3 & 1 & $1 / 2$ & $1 / 2$ & $1 / 4$ & $1 / 4$ & 0.0939 \\
\hline & 0.088 & 3 & 3 & 2 & 1 & $1 / 2$ & $1 / 4$ & $1 / 3$ & 0.1036 \\
\hline & 0.125 & 2 & 3 & 2 & 2 & 1 & $1 / 3$ & $1 / 4$ & 0.1214 \\
\hline & 1625 & 3 & 3 & 4 & 4 & 3 & 1 & $1 / 3$ & 0.2339 \\
\hline$l p_{2}$ & 0.2 & 7 & 3 & 4 & 3 & 4 & 3 & 1 & 0.3474 \\
\hline
\end{tabular}

Table 10 The second cluster "annual precipitation".

\begin{tabular}{|c|c|c|c|c|c|c|c|c|c|}
\hline & & $l p_{2}(0.2)$ & 0.3 & $l p_{3}(0.4)$ & 0.45 & $l p_{4}(0.5)$ & 0.75 & 1 & Local priority \\
\hline \multirow[t]{2}{*}{$l p_{2}$} & 0.2 & 1 & $1 / 4$ & $1 / 5$ & $1 / 3$ & $1 / 2$ & $1 / 6$ & $1 / 6$ & 0.0337 \\
\hline & 0.3 & 4 & 1 & $1 / 3$ & $1 / 4$ & $1 / 3$ & $1 / 3$ & $1 / 4$ & 0.0586 \\
\hline \multirow[t]{2}{*}{$l p_{3}$} & 0.4 & 5 & 3 & 1 & $1 / 2$ & $1 / 2$ & $1 / 4$ & $1 / 2$ & 0.0102 \\
\hline & 0.45 & 3 & 4 & 2 & 1 & $1 / 2$ & $1 / 2$ & $1 / 5$ & 0.1147 \\
\hline \multirow[t]{3}{*}{$l p_{4}$} & 0.5 & 2 & 3 & 2 & 2 & 1 & $1 / 3$ & $1 / 4$ & 0.1230 \\
\hline & 0.75 & 6 & 3 & 4 & 2 & 3 & 1 & $1 / 3$ & 0.2204 \\
\hline & 1 & 6 & 4 & 2 & 5 & 4 & 3 & 1 & 0.3479 \\
\hline
\end{tabular}

In this step, the data of each city in Hubei Province is substituted into each indicator, which is also divided into different levels. The global priority of each limiting profile and each alternative are calculated through Eqs. 27) 28, and then classified.

According to the weight method of the combination of subjective and objective, combined with the classification of AHPSort II, the water resources security level of each city in Hubei Province is obtained. Wuhan, Huangshi, Xiangyang, Ezhou, Jingmen, Xiaogan, Jingzhou, Suizhou, Xiantao, Qianjiang, and Tianmen are at high-risk; Huanggang, Xianning are at medium-high-risk; Shiyan is at medium-risk; Yichang and Shennongjia are at medium-low-risk; Enshi is at low-risk. The study area and its classification are shown in Fig. 7. The water resource security level of each city is greatly affected by the efficiency of water use.

Fig. 7 Study area. 
Table 11 Normalized local priorities of the representative points and the limiting profiles for the "annual precipitation" .

\begin{tabular}{cccc}
\hline & Representative point & $\begin{array}{c}\text { Local priority with the first cluster } \\
\text { class as the standard }\end{array}$ & Normalized local priorities \\
\hline & 0.0000 & 0.0403 & 0.0112 \\
$l p_{1}$ & 0.0250 & 0.0568 & 0.0158 \\
& 0.5000 & 0.0939 & 0.0262 \\
& 0.0880 & 0.1036 & 0.0289 \\
$l p_{2}$ & 0.1250 & 0.1214 & 0.0339 \\
& 0.1625 & 0.2339 & 0.0652 \\
$l p_{3}$ & 0.2000 & 0.3474 & 0.0969 \\
& 0.3000 & 0.6041 & 0.1684 \\
$l p_{4}$ & 0.4000 & 1.0494 & 0.2926 \\
& 0.4500 & 1.1824 & 0.3297 \\
& 0.5000 & 1.2680 & 0.3536 \\
& 0.7500 & 2.2720 & 0.6335 \\
& 1.0000 & 3.5864 & 1.0000 \\
\hline
\end{tabular}

\subsection{Comparison analysis}

In order to verify the effectiveness of the method, this paper will use the subjective weight method combined with AHPSort II and the objective subjective method combined with AHPSort II respectively, and compare with the classification obtained by the subjective and objective methods combined with AHPSort II. The comparison results are shown in Table 12 .

Table 12 Analysis results and comparative analysis.

\begin{tabular}{lcccccc}
\hline \multirow{2}{*}{ City } & \multicolumn{2}{c}{ Fuzzy AHPSort II-EW method } & \multicolumn{2}{c}{ AHPSort II-EW method } & \multicolumn{2}{c}{ AHPSort II-fuzzy method } \\
\cline { 2 - 7 } & Ra1 & Rank by Ra1 & Ra2 & Rank by Ra2 & Ra3 & Rank by Ra3 \\
\hline Wuhan & 0.1771 & V & 0.2054 & V & 0.1492 & IV \\
Huangshi & 0.0747 & V & 0.0677 & V & 0.0817 & V \\
Shiyan & 0.4014 & III & 0.3806 & IV & 0.4222 & I \\
Yichang & 0.4693 & II & 0.4679 & III & 0.4708 & I \\
Xiangyang & 0.1026 & V & 0.1021 & V & 0.1029 & V \\
Ezhou & 0.1331 & V & 0.1662 & V & 0.0999 & V \\
Jinmen & 0.0721 & V & 0.0786 & V & 0.0656 & V \\
Xiaogan & 0.0647 & V & 0.0721 & V & 0.0574 & V \\
Jinzhou & 0.1803 & V & 0.1662 & V & 0.1942 & IV \\
Huanggang & 0.2485 & IV & 0.2051 & V & 0.2915 & III \\
Xianning & 0.3001 & IV & 0.2244 & V & 0.3756 & I \\
Suizhou & 0.1208 & V & 0.1680 & V & 0.0741 & V \\
Enshi & 0.7058 & I & 0.6591 & I & 0.7520 & I \\
Xiantao & 0.1038 & V & 0.1160 & V & 0.0882 & V \\
Qianjiang & 0.1207 & V & 0.1551 & V & 0.0864 & V \\
Tianmen & 0.1191 & V & 0.1655 & V & 0.0728 & V \\
Shennongjia & 0.4706 & II & 0.4624 & III & 0.4788 & I \\
\hline
\end{tabular}

From the data in the table, it can be seen that most of the high-risk groups have no change, and a small number of groups have changes in high-risk. Moreover, the combination of subjective and objective methods is the neutralization result of the other two methods. The common level areas obtained by the three methods often show many or few indicators higher than the limit conditions, which also shows that most of the results calculated by the three methods are not very different, which also shows that this method is a fine adjustment of the results of the other two methods and has better reliability. The following also talks about the disadvantages of using only one weight method alone.

As far as the entropy weight method is concerned, it is a typical objective weight calculation method. It also contains some disadvantages of other objective weight methods. It depends on enough sample data and 

in practical problems also needs to be considered.

Fig. 8 The influence of $V$ on indicators weight.

Fig. 9 The influence of $V$ on limiting profile.

actual problem domain. Sometimes, the resulting weights will be very different from the actual importance of the attribute. Therefore, it is necessary to combine this method with experts' evaluations. For example, as far as precipitation is concerned, the precipitation distribution in Hubei Province is uneven. Some cities have a lot of precipitation, while others don't. In terms of data, it may be that cities with more precipitation are much better than those with less precipitation. But in fact, experts may think that this indicator is not so important, which will cause errors.

The expert scoring method is a subjective method, but because each expert's work experience and educational background are not the same, It will lead to divergence among each expert. Although, this paper uses a model to make the consensus of each expert reach the highest and the divergence reach the minimum, it is difficult for experts to reach complete agreement, it would be better if there were an objective method to supplement.

In a word, if only one subjective or objective method is used to calculate the weight of indicators, it is unreliable, and the combination of the two methods can get more reliable results.

\subsection{Sensitivity Analysis}

The weight method in this paper is based on the combination of entropy weight method and hesitant fuzzy language judgment. There is a parameter $V$ in the formula of combination weight, whose value is between 0 and 1. Therefore, it is necessary to test the influence of different values of $V$ on the final weight result. When $v=0$, it means that the only subjective method is used to get the weight, and when $V=1$, it means the only objective method is used to get the weight Weight. Fig. 8 shows that the weight of each indicator changes with the change of parameter $V$. It can be seen that there are criterions with great changes in each indicator. Some indicators increase with the change of $V$, while others decrease. $C_{33}$ and $C_{34}$ are almost the same under the efficiency of water use, while $C_{32}$ shows a great growth trend. The change of indicator weight leads to the change of global priority and global limiting profile, and finally affects the security level of each alternative. Through Fig. 9 it is not difficult to find that with the increasing of $V$, the security level of some alternatives near each of these global limiting profiles will change. It can be seen from Table 13 that the final evaluation grade of many cities does not change due to the change of $V$, but the overall change is towards a safer grade with the increase of $V$. In general, the whole data does not show a large span change with the slight change of $V$, therefore, it is necessary to use the subjective and objective methods to calculate the weight at the same time, and the determination of parameters in this link needs to be careful, and the deviation caused by parameter selection

\subsection{Suggestions}

It can be seen from Table 3 that the distribution of water resources in various cities of Hubei Province is very uneven. In addition, Hubei Province is also a strong provincial capital province. As the provincial capital, Wuhan is the largest city in the province, which has a big gap with the second-ranked city. Therefore, it can be seen that Wuhan, as the provincial capital, has a gap with other cities in many indicators. It can be seen from Table 12 that most of the cities in Hubei Province are at a dangerous level. As a province with many water systems, the security of water resources in Hubei Province is mainly the pollution of water resources 
Table 13 The influence of $V$ on the security level.

\begin{tabular}{|c|c|c|c|c|c|c|c|c|c|c|c|}
\hline City & $V=0$ & $V=0.1$ & $V=0.2$ & $V=0.3$ & $V=0.4$ & $V=0.5$ & $V=0.6$ & $V=0.7$ & $V=0.8$ & $V=0.9$ & $V=1$ \\
\hline Wuhan & $\mathrm{V}$ & $\mathrm{V}$ & $\mathrm{V}$ & $\mathrm{V}$ & $\mathrm{V}$ & $\mathrm{V}$ & $\mathrm{V}$ & IV & IV & IV & IV \\
\hline Huangshi & $\mathrm{V}$ & $\mathrm{V}$ & $\mathrm{V}$ & $\mathrm{V}$ & $\mathrm{V}$ & $\mathrm{V}$ & $\mathrm{V}$ & $\mathrm{V}$ & $\mathrm{V}$ & $\mathrm{V}$ & $\mathrm{V}$ \\
\hline Shiyan & IV & III & III & III & III & III & III & $\mathrm{V}$ & $\mathrm{V}$ & IV & IV \\
\hline Yichang & III & III & III & III & III & II & II & II & II & II & I \\
\hline Xiangyang & $\mathrm{V}$ & $\mathrm{V}$ & $\mathrm{V}$ & $\mathrm{V}$ & $\mathrm{V}$ & $\mathrm{V}$ & $\mathrm{V}$ & $\mathrm{V}$ & $\mathrm{V}$ & $\mathrm{V}$ & $\mathrm{V}$ \\
\hline Ezhou & $\mathrm{V}$ & $\mathrm{V}$ & $\mathrm{V}$ & $\mathrm{V}$ & $\mathrm{V}$ & $\mathrm{V}$ & $\mathrm{V}$ & $\mathrm{V}$ & $\mathrm{V}$ & $\mathrm{V}$ & $\mathrm{V}$ \\
\hline Jinmen & $\mathrm{V}$ & $\mathrm{V}$ & $\mathrm{V}$ & $\mathrm{V}$ & $\mathrm{V}$ & $\mathrm{V}$ & $\mathrm{V}$ & $\mathrm{V}$ & $\mathrm{V}$ & $\mathrm{V}$ & $\mathrm{V}$ \\
\hline Xiaogan & $\mathrm{V}$ & $\mathrm{V}$ & $\mathrm{V}$ & $\mathrm{V}$ & V & $\mathrm{V}$ & $\mathrm{V}$ & $\mathrm{V}$ & V & $\mathrm{V}$ & $\mathrm{V}$ \\
\hline Jinzhou & $\mathrm{V}$ & $\mathrm{V}$ & $\mathrm{V}$ & $\mathrm{V}$ & $\mathrm{V}$ & $\mathrm{V}$ & $\mathrm{V}$ & IV & IV & IV & IV \\
\hline Huanggang & IV & IV & IV & IV & IV & III & III & III & III & III & III \\
\hline Xianning & $\mathrm{V}$ & $\mathrm{V}$ & IV & IV & IV & IV & III & III & III & III & II \\
\hline Suizhou & $\mathrm{V}$ & $\mathrm{V}$ & $\mathrm{V}$ & $\mathrm{V}$ & $\mathrm{V}$ & $\mathrm{V}$ & $\mathrm{V}$ & $\mathrm{V}$ & $\mathrm{V}$ & $\mathrm{V}$ & $\mathrm{V}$ \\
\hline Enshi & II & I & I & I & I & I & I & I & I & I & I \\
\hline Xiantao & $\mathrm{V}$ & $\mathrm{V}$ & $\mathrm{V}$ & $\mathrm{V}$ & V & $\mathrm{V}$ & $\mathrm{V}$ & $\mathrm{V}$ & $\mathrm{V}$ & $\mathrm{V}$ & V \\
\hline Qianjiang & $\mathrm{V}$ & $\mathrm{V}$ & $\mathrm{V}$ & $\mathrm{V}$ & $\mathrm{V}$ & $\mathrm{V}$ & $\mathrm{V}$ & $\mathrm{V}$ & $\mathrm{V}$ & $\mathrm{V}$ & $\mathrm{V}$ \\
\hline Tianmen & $\mathrm{V}$ & $\mathrm{V}$ & $\mathrm{V}$ & $\mathrm{V}$ & $\mathrm{V}$ & $\mathrm{V}$ & $\mathrm{V}$ & $\mathrm{V}$ & $\mathrm{V}$ & $\mathrm{V}$ & $\mathrm{V}$ \\
\hline Shennongjia & III & III & III & III & III & II & II & II & II & II & I \\
\hline
\end{tabular}

and the efficiency of water use. At present, agricultural irrigation accounts for $67.16 \%$, industry accounts for $15.13 \%$ and rural life accounts for $20 \%$ of the total water consumption of the province $12 \%$, forestry, animal husbandry and fishing accounted for $5.13 \%$, and urban life accounted for $2.16 \%$. From the perspective of water consumption structure, agricultural irrigation accounts for the majority of water consumption, and there is great potential for agricultural water saving. In terms of farmland irrigation in Hubei Province, flood irrigation is still very common, and the utilization rate of diversion canal is only $50 \%$, that is, half of the water is leaked and evaporated. In addition, the reuse rate of industrial water in Hubei Province is very low, about $50 \%$ in Wuhan and only $20 \%$ - $40 \%$ in other cities, therefore, by introducing the indicators of water resource use efficiency, we can see that most cities in Hubei Province are at a high risk level, which is different from our previous cognition of Hubei Province and deserves our attention to water resource security in areas with abundant water resource. By introducing the index of water resource use efficiency, we can see that most cities in Hubei province are at a high risk level, which is different from our previous cognition of Hubei Province and deserves our attention to water resource security in water-rich areas. And some cities in the middle-risk and low-risk level are basically because they are sparsely populated, have a good ecological environment, and have a low economy. There is little water consumption in all aspects, such as Enshi. Yichang's economy is at the forefront of the province, and its population ranks sixth among the 17 cities in the province, but the safety of water resources is at low risk because the existence of the Three Gorges Dam makes the use efficiency of water resources very high. This paper proposes the following methods to deal with this phenomenon:

(1) In agriculture, Hubei Province still uses flood irrigation and has a low industrial water reuse rate. In view of the above two points, the following improvements can be made. In agriculture, water resource fees and fees can be levied, quota allocation can be implemented, and water-saving agricultural technology irrigation can be promoted, especially in the dry land areas of northern Hubei Province. In the industry, we can realize water quota allocation by collecting water fees and water, the full implementation of social water standard quota, and improve water quality, to encourage water-saving and clean production, improve the utilization rate of water and reduce water consumption per unit product, in the city life water use should gradually improve the sewage disposal, and improve the public awareness of water through the publicity.

(2) About water pollution has always been the focus of the water security problem, so the water quality of the recovery will take various efforts, in the first place to have a scientific and reasonable method to control water pollution and secondly from the source control water pollution is to reduce the emissions of pollutants, the solution to water pollution cannot be copied elsewhere, Because the levels of pollution that each place faces 
and the causes of pollution are different, it is necessary to identify the effects and targets of the final effects during the planning process.

(3) Not only in Hubei Province, but the efficiency of water resources also use in the whole of China still needs to be improved. Although water resources in Hubei Province are relatively abundant in China, due to the uneven distribution, how to rationally use water resources to improve the efficiency of water resources use becomes particularly important. Virtual water strategy is a new direction in water resources research, and also a new practical direction in water resources management. And this is certainly a good way to solve the problem.

\section{Conclusions and Future Research Direction}

This paper argues that water resources are indispensable to human beings' social production and life, and it is particularly important to ensure the security of water resources. Based on the above analysis, this paper proposes a novel method to evaluate the security of regional water resources.

Firstly, as a matter of fact, there are many provinces in China with abundant water resources, however the distribution of water resources is uneven and the use efficiency of water resources is low. Therefore, in addition to considering two criteria of quantity of water resources and pressure on water resources, this paper emphatically takes the criterion of efficiency of water use into account, all these are based on previous related studies and practical problems. This paper mainly explores a water resource security evaluation system which is suitable for the regions with abundant water resources but uneven distribution and low water resource utilization efficiency. As virtual water can better embody water efficiency utilization efficiency, this paper introduces virtual water as well as visible water to evaluate the security of water resources.

Secondly, this paper combines subjective weight and objective weight to get indicators' weights. For the subjective method, hesitant fuzzy language term sets are employed to describe the experts' judgements, then the model with the minimum disagreement and the maximum consensus is applied to obtain the experts' weights, the indicators' weights is calculated by introducing the parameters of weighted average operator. For the objective method, the entropy weight is utilized, which is adjusted by the subjective method. Furthermore, the combined indicators' weights are substituted into fuzzy AHPSort II-EW method to classify the alternatives, which improves the fairness and reliability of the evaluation results.

Thirdly, the proposed method is applied to evaluate the water security of 17 cities of Hubei Province in 2019. Hubei Province, as a province with abundant water resource but inefficient in the use of water resources and uneven distribution of water resources. The water resources security of Hubei Province is divided into five levels with 11 cities at high-risk level, 2 cites at medium-high-risk level, 1 city at medium-risk level, 2 cities at medium-low-risk level, and 1 city at low-risk level.

Finally, the comparison of AHPSort II-EW method, and AHPSort II-fuzzy method shows that the proposed fuzzy AHPSort II-EW method method which considers both subjective and objective weights is more reliable. In addition, sensitivity analysis is also carried out on the parameter $V$ of combined weight, which demonstrates the efficiency of the subjective/objective combined weight method. Based on above analysis, some suggestions are given. As Hubei Province well-water resourced region, the shocked result is a reminder of water resources use efficiency that affect water security in areas with abundant water resources.

The proposed method used in this paper can also be applied to the evaluation of water resources security in other provinces or regions, especially in areas with sufficient water resources but low efficiency and uneven distribution of water resources. The limitations of this paper and the areas that can be further studied in the future include the following aspects: This paper only uses an entropy weight method as an objective weight calculation method. In the future, the research direction of the method can choose the weight calculation method according to the actual problems of the research. This paper does not consider the flood factor in determining water resources security. In the future, the research direction of water resources security can add research objects. 


\section{Declarations}

Ethics approval and consent to participate

Not applicable.

\section{Consent to Publish}

Not applicable.

Authors' contributions

YD: Data curation, Formal analysis, Investigation, Writing - original draft; YT: Methodology, Writing review \& editing; ZL: Writing - review \& editing; LN: Formal analysis.

Funding

This research was supported by the National Natural Science Foundation of China (grant number 71801177), the Humanities and Social Sciences Fund of Ministry of Education of China (grant number 18YJC630163), and the Fundamental Research Funds for the Central Universities (grant number WUT: 2020VI006).

\section{Competing interests}

The authors declare that they have no conflict of interest.

Availability of data and materials

The datasets used and/or analysed during the current study are available from the corresponding author on reasonable request.

\section{References}

Aguaron J, Escobar MT, Moreno-Jimenez JM, Turon A (2019) AHP-Group decision making based on consistency. Mathematics $7(3): 242$. https://doi.org/10.3390/math7030242

Acuna-Alonso C, Fernandes ACP, Alvarez X, Valero E, Pacheco FAL, Varandas SDP, Terencio DPS, Fernandes LFS (2021) Water security and watershed management assessed through the modelling of hydrology and ecological integrity: A study in the Galicia-Costa (NW Spain). Science of the Total Environment 759:143905. https://doi.org/10.1016/j.scitotenv.2020.143905

Abbott BW, Bishop K, Zarnetske JP, Minaudo C, Chapin FS, Krause S, Hannah DM, Conner L, Ellison D, Godsey SE, Plont S, Marcais J, Kolbe T, Huebner A, Frei RJ, Hampton T, Gu S, Buhman M, Sayedi SS, Ursache O, Chapin M, Henderson KD, Pinay G (2019) Human domination of the global water cycle absent from depictions and perceptions. Nature Geoscience 12(7):533-+. https://doi.org/10.1038/s41561-019-0374-y

Cao XC, Zeng W, Wu MY, Li TY, Chen S, Wang WG (2021) Water resources efficiency assessment in crop production from the perspective of water footprint. Journal of Cleaner Production 309:0959-6526. https://doi.org/10.1016/j.jclepro.2021.127371

Chen WM, Kang JN, Han MS (2021) Global environmental inequality: Evidence from embodied land and virtual water trade. Science of the Total Environment 783:146992. https://doi.org/10.1016/j.scitotenv.2021.146992

de Melo MC, Formiga-Johnsson RM, de Azevedo JPS, Nascimento ND, Machado FLV, Pacheco FAL, Fernandes LFS (2021) A raw water security risk model for urban supply based on failure mode analysis. Journal of Hydrology $593: 125843$. https://doi.org/10.1016/j.jhydrol.2020.125843

de Graaf IEM. Gleeson T, van Beek LPH, Sutanudjaja EH, Bierkens MFP (2019) Environmental flow limits to global groundwater pumping. Nature: International weekly journal of science 574(7776):90-+. https://doi.org/10.1038/s41586-019-1594-4 
Ding XW, Chong X, Bao ZF, Xue Y, Zhang SH (2017) Fuzzy comprehensive assessment method based on the entropy weight method and its application in the water environmental safety evaluation of the Heshangshan drinking water source area, Three Gorges reservoir area, China. Water 9(5):329. https://doi.org/10.3390/w9050329

Dou PF, Zuo SD, Ren Y, Rodriguez MJ, Dai SQ (2021) Refined water security assessment for sustainable water management: A case study of 15 key cities in the Yangtze River Delta, China. Journal of Environmental Management $290: 112588$. https://doi.org/10.1016/j.jenvman.2021.112588

Fu YP, Huang GH, Liu LR, Li JY, Zhang XY, Zhai MY, Pan XJ (2021) Multi-hierarchy virtual-water management; A case study of Hubei Province, China. Journal of Cleaner Production 293:126244. https://doi.org/10.1016/j.jclepro.2021.126244

Gusain R, Kumar N, Ray SS (2020) Recent advances in carbon nanomaterial-based adsorbents for water purification. Coordination Chemistry Reviews 405:213111. https://doi.org/10.1016/j.ccr.2019.213111

Hubei Water Resources Bulletin in 2019. http://slt.hubei.gov.cn/

Ishizaka A, Lopez C (2018) Cost-benefit AHPSort for performance analysis of offshore providers. International Journal of Production Research 57(13):4261-4277.https://doi.org/10.1080/00207543.2018.1509393

Kang HY, Lee AHI, Chan YC (2019) An integrated fuzzy multi-criteria decision-making approach for evaluating business process information systems. Mathematics 7(10):982. https://doi.org/10.3390/math7100982

Kang SZ, Hao XM, Du TS, Tong L, Su XL, Lu HN, Li XL, Huo ZL, Li SE, Ding RS (2017) Improving agricultural water productivity to ensure food security in China under changing environment: From research to practice. Agricultural Water Management 179:5-17. https://doi.org/10.1016/j.agwat.2016.05.007

Li B, Wu Q, Zhang WP, Liu ZJ (2020) Water resources security evaluation model based on grey relational analysis and analytic network process: A case study of Guizhou Province. Journal of Water Process Engineering 37:101429. https://doi.org/10.1016/j.jwpe.2020.101429

Li, ZM, Zhang Q, Liao, HC (2018) Efficient-equitable-ecological evaluation of regional water resource coordination considering both visible and virtual water. Omega 83:223-235. https://doi.org/10.1016/j.omega.2018.06.003

Liu YL, Zhuo L, Varis O, Fang K, Liu G, Wu P (2021) Enhancing water and land efficiency in agricultural production and trade between Central Asia and China. Science of the Total Environment 780:146584. https://doi.org/10.1016/j.scitotenv.2021.146584

Li ZM, Xu JP, Lev B, Gang J (2015) Multi-criteria group individual research output evaluation based on context-free grammar judgments with assessing attitude. Omega 57:282-293 B. https://doi.org/10.1016/j.omega.2015.09.001

Luo Y, Chen XD, Yao LM (2020) Flood disaster resilience evaluation of Chinese regions: integrating the hesitant fuzzy linguistic term sets with prospect theory. Natural Hazards 105(1):667-690. https://doi.org/10.1007/s11069-020-04330-z

Liu ZH, Jiang ZZJ, Xu C, Cai GJ, Zhan J (2021) Assessment of provincial waterlogging risk based on entropy weight TOPSIS-PCA method. Natural Hazards 108(2):1545-1567. https://doi.org/10.1007/s11069-021-04744-3

Luo PP, Sun YT, Wang ST, Wang SM, Lyu JQ, Zhou MM, Nakagami K, Takara k, Nover D (2020) Historical assessment and future sustainability challenges of Egyptian water resources management. Journal of Cleaner Production 263:121154. https://doi.org/10.1016/j.jclepro.2020.121154

Ministry of Environmental Protection of China. Report on the State of the Environment in China (2015); Environmental Information Centre, Ministry of Environmental Protection of the People's Republic of China: Beijing, China, 2015

Mishra, V (2020) Long-term (1870-2018) drought reconstruction in context of surface water security in India. Journal of Hydrology 580:124228. https://doi.org/10.1016/j.jhydrol.2019.124228

Miccoli F, Ishizaka A (2017) Sorting municipalities in Umbria according to the risk of wolf attacks with AHPSort II. Ecological Indicators 73:741-755. https://doi.org/10.1016/j.ecolind.2016.10.034

Meng FY, Tang J (2019) New ranking order for linguistic hesitant fuzzy sets. J. Oper. Res. Soc 70:531-540. https://doi.org/10.1080/01605682.2018.1447252

Nilsson M, Griggs D, Visbeck M (2016) Map the interactions between Sustainable Development Goals. Nature 534(7607):320-322. https://doi.org/10.1038/534320a

National Bureau of statistics. http://www.stats.gov.cn/

Sun C, Li SY, Deng Y (2020) Determining weights in multi-criteria decision making based on negation of probability distribution under uncertain environment. Mathematics 8(2):191. https://doi.org/10.3390/math8020191

Sutadian AD, Muttil N, Yilmaz AG, Perera BJC (2017) Using the analytic hierarchy process to identify parameter weights for developing a water quality index. Ecological Indicators 75:220-233. https://doi.org/10.1016/j.ecolind.2016.12.043

Song ML, Zhu QY, Peng J, Gonzalez EDRS (2017) Improving the evaluation of cross efficiencies: A method based on Shannon entropy weight. Computers \& Industrial Engineering 112:99-106. https://doi.org/10.1016/j.cie.2017.07.023

Tu Y, Wang HY, Zhou XY, Shen WJ, Lev B, Klemes JJ (2021) Comprehensive evaluation of security, equity, and efficiency on regional water resources coordination using a hybrid multi-criteria decision-making method with different hesitant fuzzy linguistic term sets. Journal of Cleaner Production 310:127447. https://doi.org/10.1016/j.jclepro.2021.127447

The Ministry of Water Resources releases 2019 China Water Resources Bulletin. https://doi:CNKI:SUN:SJYF.0.2020-09-018.

The United Nations World Water Development Report 2019. https://https://unhabitat.org/world-water-development-report-2019

Vorosmarty CJ, McIntyre P B, Gessner M O, Dudgeon D, Prusevich A, Green P, Glidden S, Bunn S E, Sullivan C A, Liermann C Reidy, Davies P M (2010) Global threats to human water security and river biodiversity. Nature 467(7315):555-561. https://doi.org/10.1038/nature.2010.09440 
Wang XX, Chen YN, Li Z, Fang GH, Wang Y (2020) Development and utilization of water resources and assessment of water security in Central Asia. Agricultural Water Management 240:106297. https://doi.org/10.1016/j.agwat.2020.106297

Xu ZW, Yao LM, Zhang Q, Dowaki K, Long Y (2020) Inequality of water allocation and policy response considering virtual water trade: A case study of Lanzhou city, China. Journal of Cleaner Production 269:122326. https://doi.org/10.1016/j.jclepro.2020.122326

Yin BL, Guan DJ, Zhou LL, Zhou J, He XJ (2020) Sensitivity assessment and simulation of water resource security in karst areas within the context of hydroclimate change. Journal of Cleaner Production 258:120994. https://doi.org/10.1016/j.jclepro.2020.120994

Xu Z, Qin JD, Liu J, Martinez L (2019) Sustainable supplier selection based on AHPSort II in interval type-2 fuzzy environment. Information Sciences 483:273-293. https://doi.org/10.1016/j.ins.2019.01.013

Yan HB, Ma TJ, Huynh VN (2016) On qualitative multi-attribute group decision making and its consensus measure: A probability based perspective. Omega 70:94-117. https://doi.org/10.1016/j.omega.2016.09.004

Zhang YX, Xu ZS, Liao HC (2019) Water security evaluation based on the TODIM method with probabilistic linguistic term sets. Soft Computing 23(15):6215-6230. https://doi.org/10.1007/s00500-018-3276-9

Zhang CQ, Wang C, Zhang ZM, Tian DZ (2019) A novel technique for multiple attribute group decision making in interval-valued hesitant fuzzy environments with incomplete weight information. Journal of Ambient Intelligence and Humanized Computing 10(6):2429-2445. https://doi.org/10.1007/s12652-018-0912-2

Zhang H, Li YP, Sun J, Liu J, Huang GH, Ding YK, Wu XJ (2021) A two-stage factorial-analysis-based input-output model for virtual-water quantification and metabolic-network identification in Kyrgyzstan. Journal of Cleaner Production 301:126960. https://doi.org/10.1016/j.jclepro.2021.126960

Zhao J, Chen YQ, Xu JC, Jin JL, Wang GQ, Shamseldin A, Guo Y, Cheng L (2021) Regional water security evaluation with risk control model and its application in Jiangsu Province, China. Environmental science and pollution research international. https://doi.org/10.1007/s11356-021-14547-5 
Figure 1

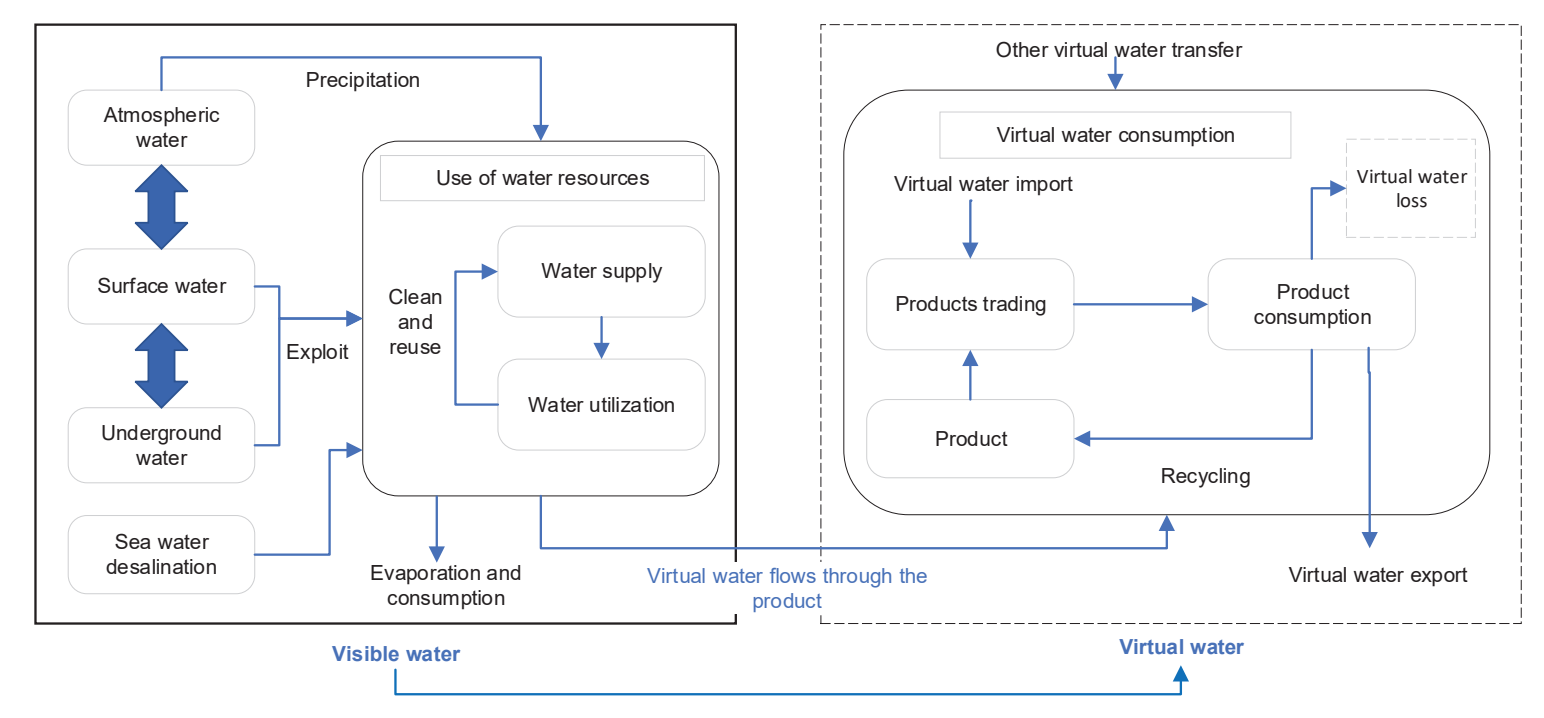

.


Figure 2

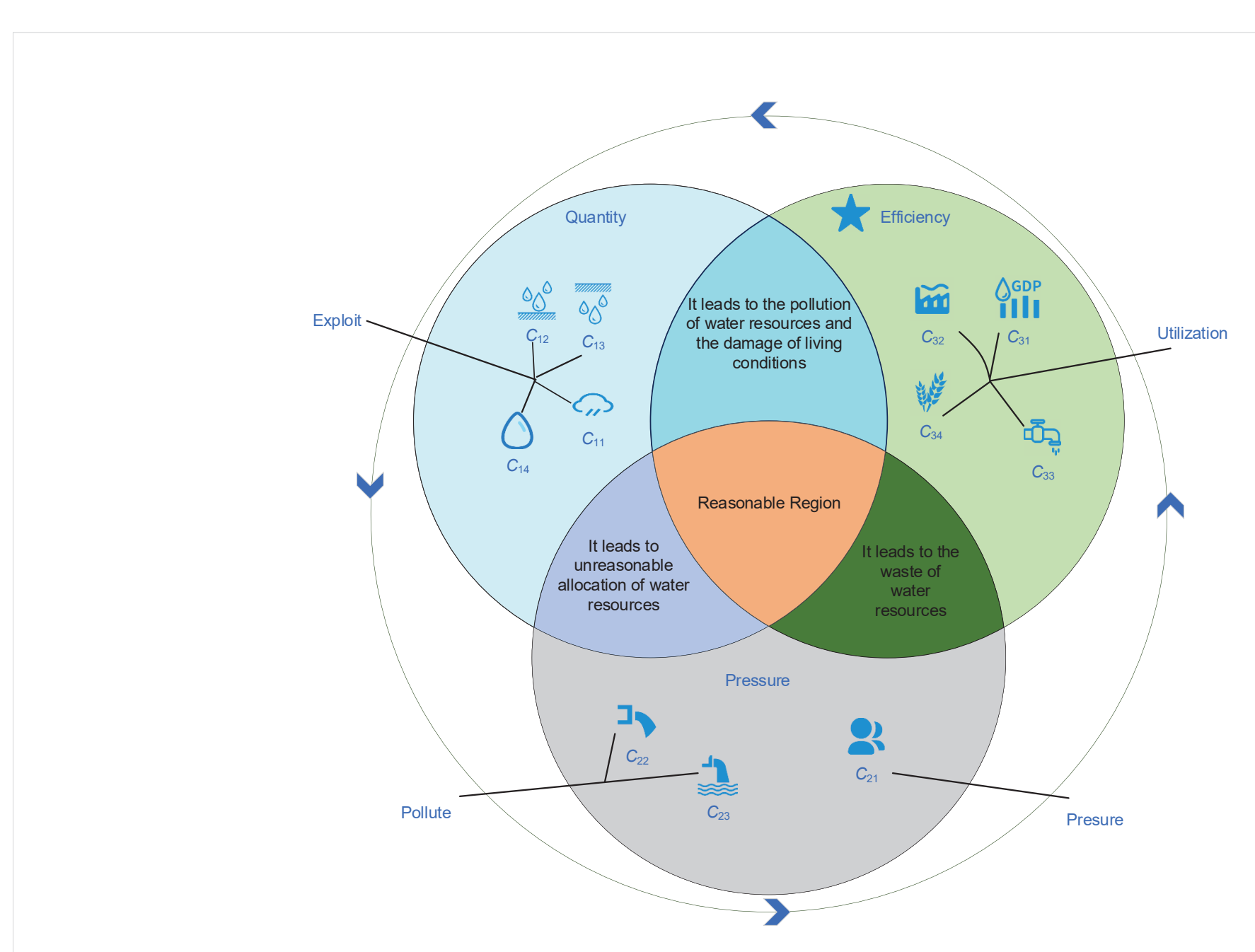

\section{.}




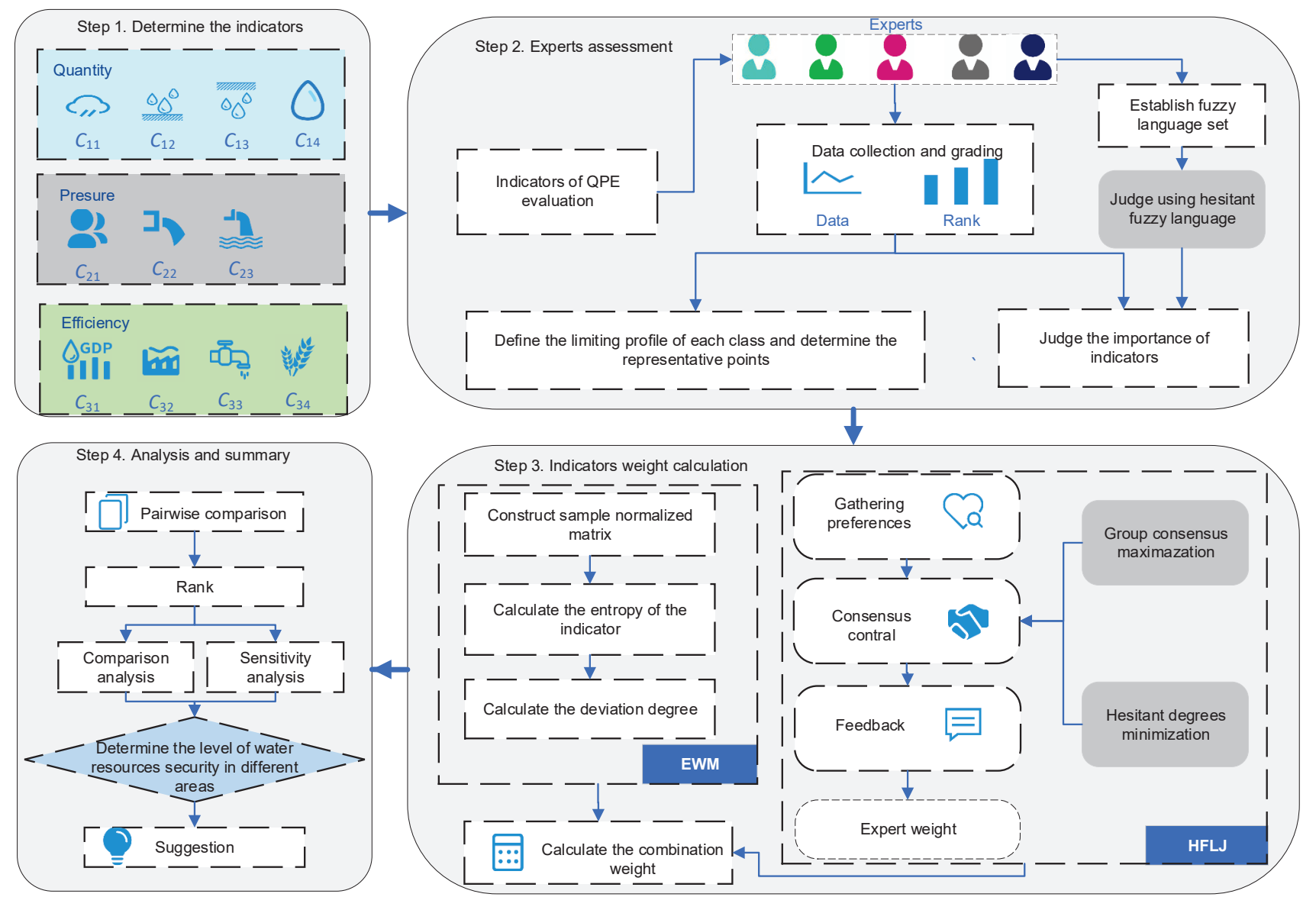


Figure 4

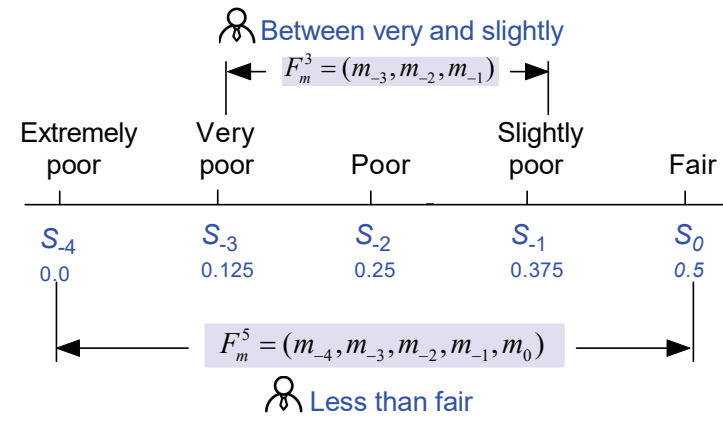

R Slightly good $\mathrm{R}$ Between good and very good $F_{m}^{1}=\left(m_{1}\right)$

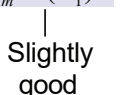

$F_{m}^{2}=\left(m_{2}, m_{3}\right)$

Very Extremely good good good

$\begin{array}{lllll}S_{1} & S_{2} & S_{3} & S_{4}\end{array}$

$F_{m}^{4}=\left(m_{1}, m_{2}, m_{3}, m_{4}\right) \longrightarrow$

B Better than slightly good 
Figure 5

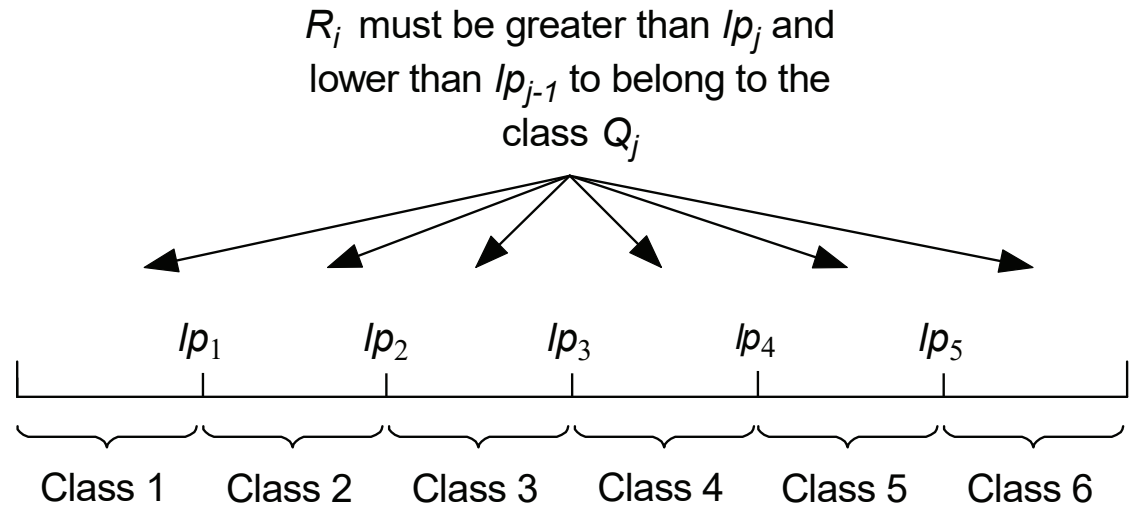

$R_{i}$ must be greater than $I p_{j}$ and class $Q$ 


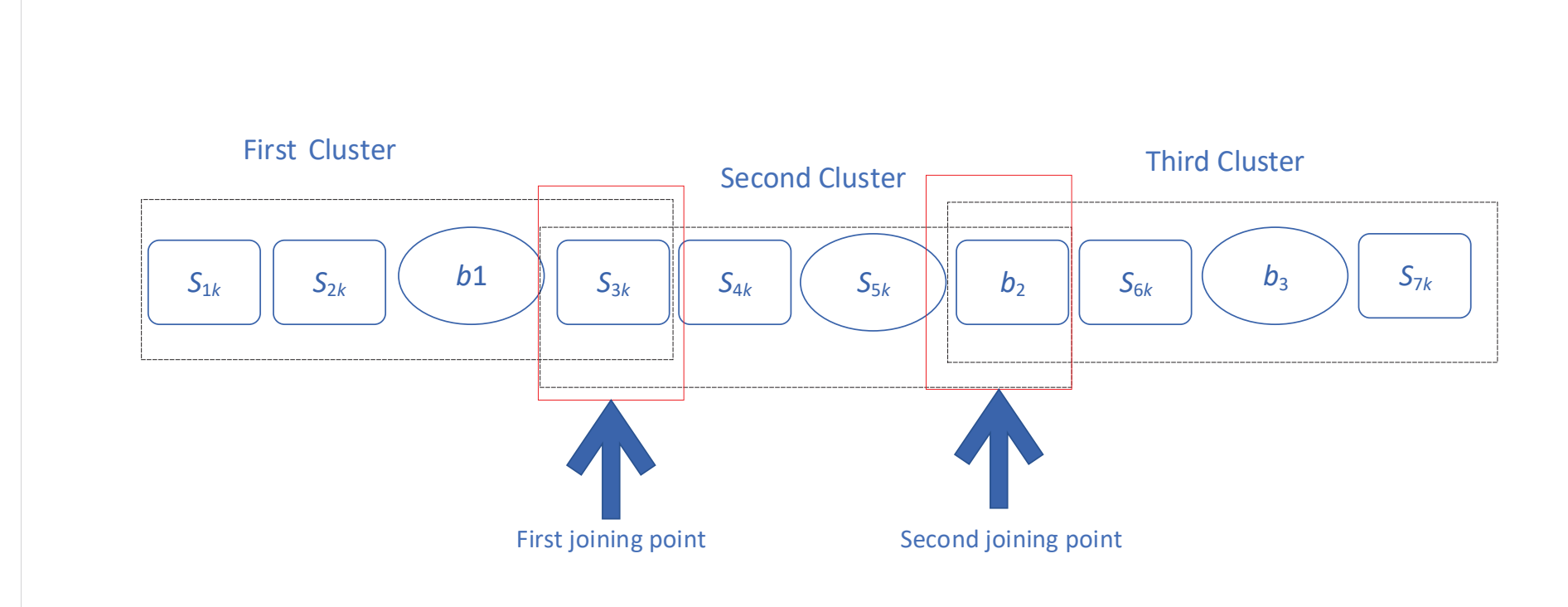

Figure 6

First joining point

Second joining point

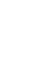

Figure 6

.

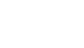

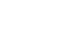

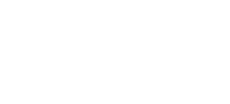

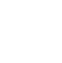

(n)

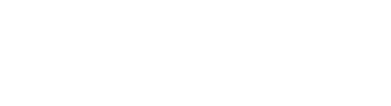

|

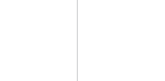

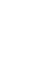

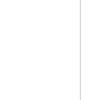

$S_{7 k}$

(1)

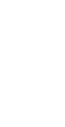




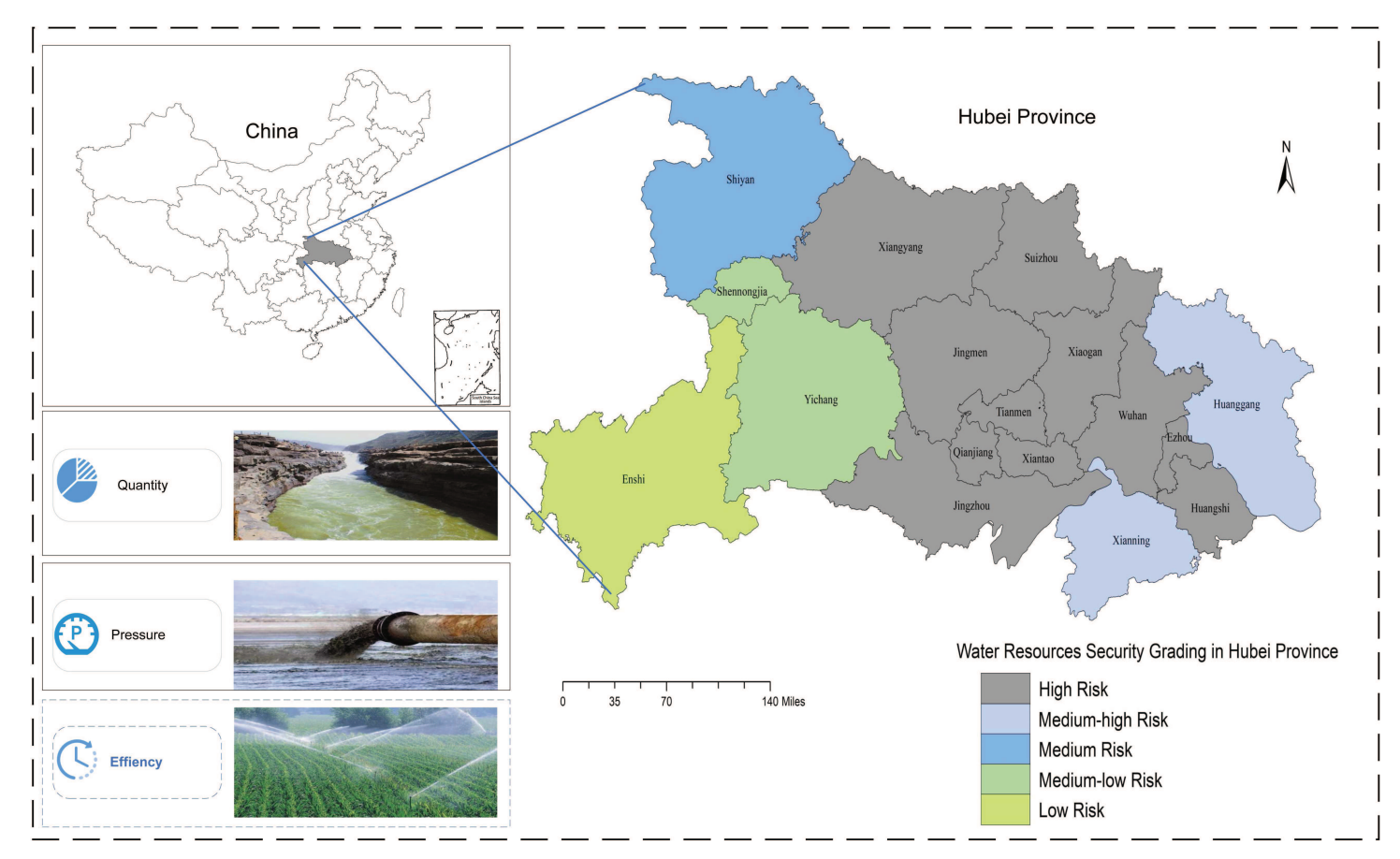

- Low Risk ------1 
Figure 8
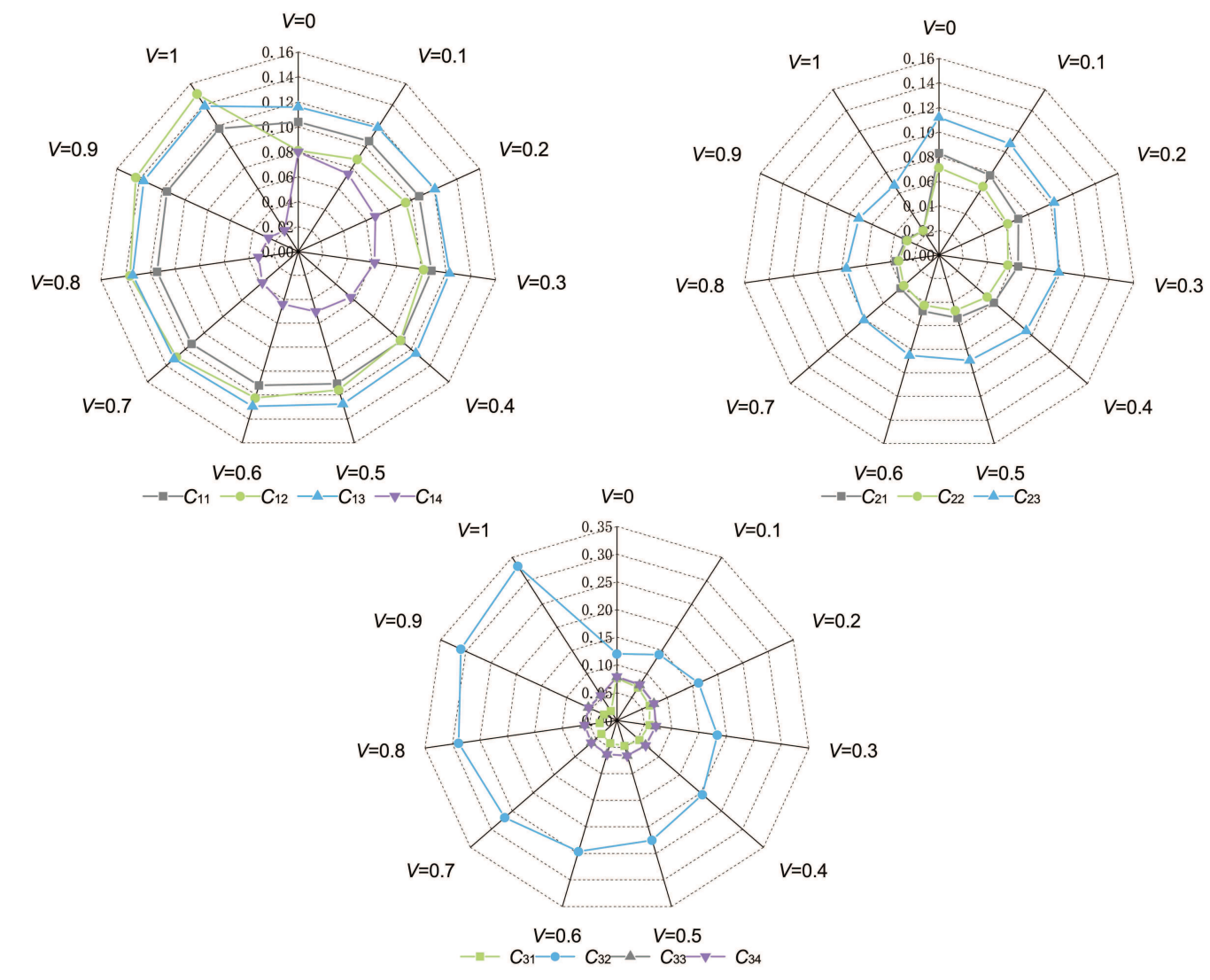
Figure 9

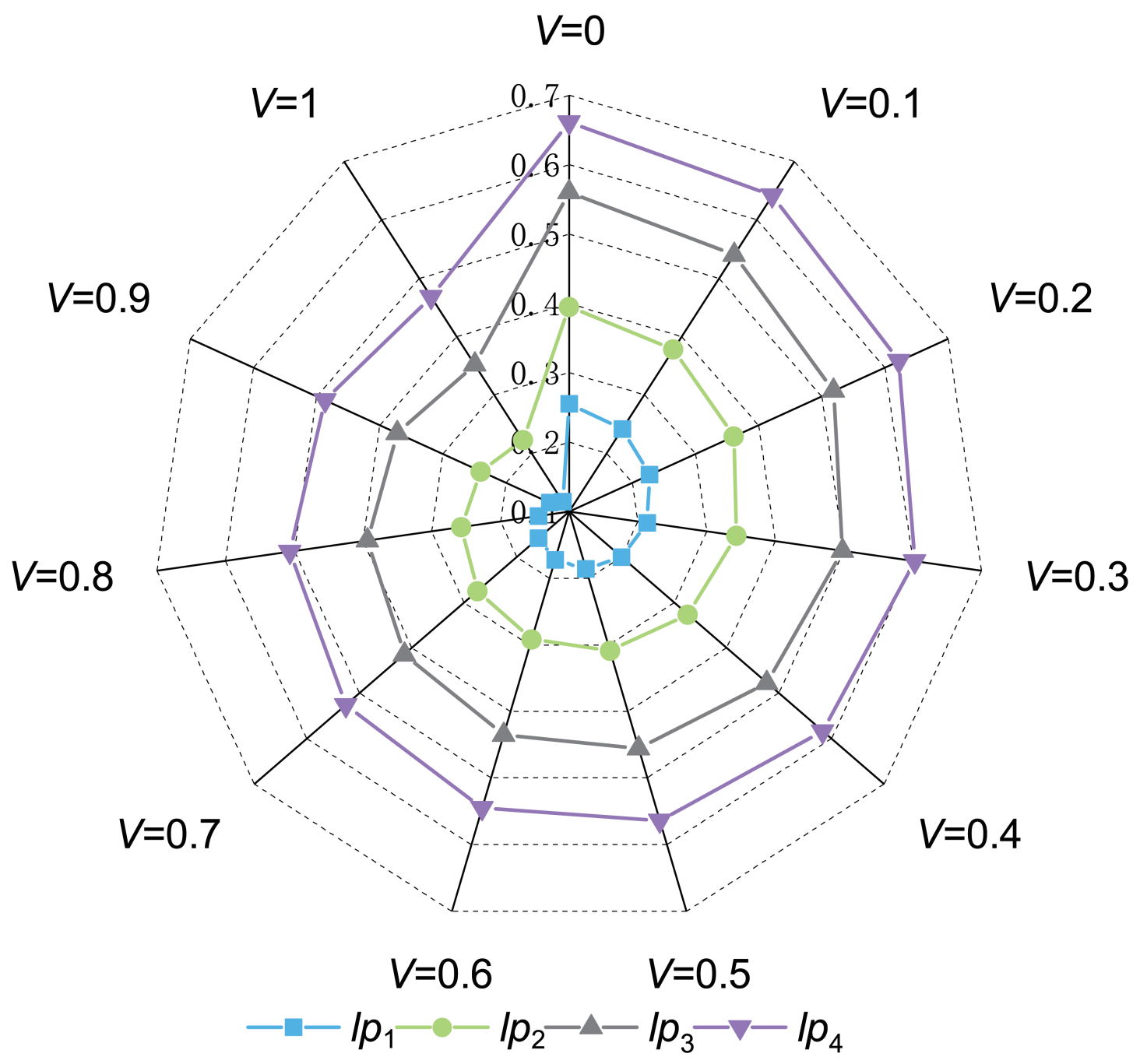

